\title{
A Design Aid for Crystal Growth Engineering
}

\author{
Jinjin Li ${ }^{\mathrm{a}, \mathrm{b}, \dagger,{ }^{*}}$, Carl J. Tilbury, ${ }^{\mathrm{b}, \dagger}$, Seung Ha Kim, ${ }^{\mathrm{b}}$ and Michael F. Doherty ${ }^{\mathrm{b},{ }^{*}}$ \\ ${ }^{a}$ National Key Laboratory of Science and Technology on Micro/Nano Fabrication, \\ Department of Micro/Nano Electronics, Shanghai Jiao Tong University, \\ Shanghai, 200240, China. \\ ${ }^{\mathrm{b}}$ Department of Chemical Engineering, University of California, Santa Barbara, California, \\ 93106, United States. \\ $\uparrow$ Contributed equally to this work \\ *E-mail: lijinjin.physics@gmail.com; $\underline{\text { mfd@engineering.ucsb.edu. }}$
}

\begin{abstract}
With the highly competitive development of chemical and pharmaceutical industries, mastering crystal growth is becoming increasingly necessary. Modern industrial manufacturers place high importance on the ability to grow crystals with a specific habit using tailored operating conditions. A detailed understanding of crystal growth is, therefore, vital for researchers in crystallography and crystallization to respond and realize this objective. Various models to predict crystal shape in the literature are reviewed here. The most commonly adopted are usually non-mechanistic and limited in their predictive power and utility, especially for products of industrial interest. Mechanistic models offer far more potential for rational crystal design, but
\end{abstract}


require significant expertise to use and each new system studied typically requires additional investment. In this context, an automated implementation of mechanistic models (simulating and visualizing crystal growth under different environmental parameters) could eliminate this barrier to entry and promote widespread adoption to propel design of crystalline-based products into the next generation. With this need in mind, we have developed prototype software named ADDICT (Advanced Design and Development of Industrial Crystallization Technology), that enables an established spiral growth model to be applied to general systems of industrial interest. This proofof-concept software provides an advanced theoretical framework to account for the solid state physics and surface chemistry, to guide experiments in a more efficient search of the design space for conditions that confer optimum functionality to the product. ADDICT calculates relative growth rates of crystal faces under the spiral regime, grown from vapor or solution, using information on the solid-state interactions that are organized into periodic bond chains. Solvent effects are principally accounted for by an interfacial modification of surface energies and the evolution of crystal habit under cycles of growth or dissolution can be predicted also. ADDICT has been tested for a variety of organic molecules, both centrosymmetric and non-centrosymmetric, resulting in successful predictions (examples for naphthalene, anthracene, paracetamol, lovastatin, D-mannitol, and $\alpha$-glycine are presented).

Keywords: crystal growth, spiral growth, periodic bond chains, kink sites and surface chemistry, mechanistic modeling. 


\section{Contents}

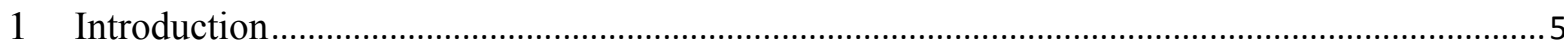

2 Non-mechanistic models for crystal habit .................................................................................

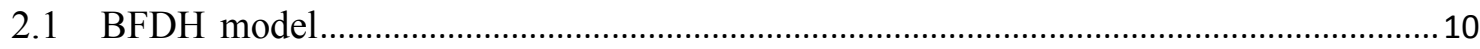

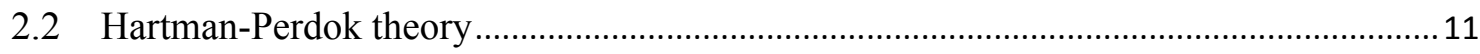

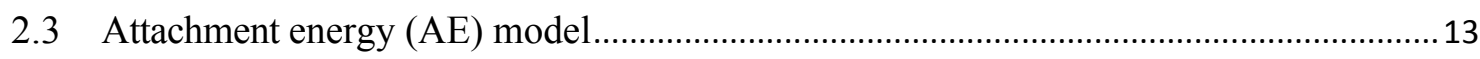

2.4 Modified attachment energy (MAE) models .............................................................. 14

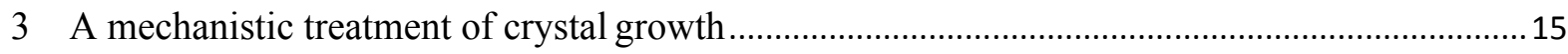

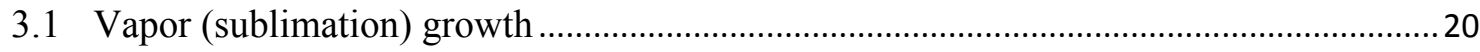

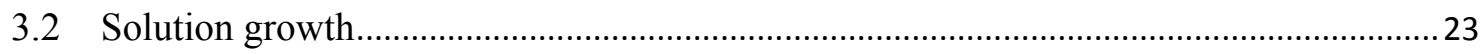

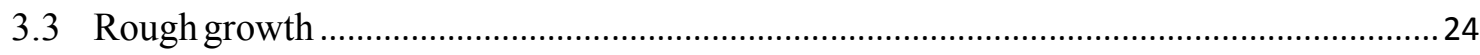

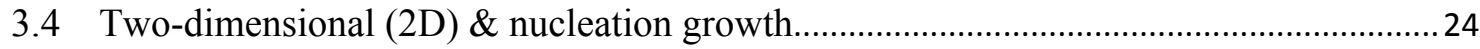

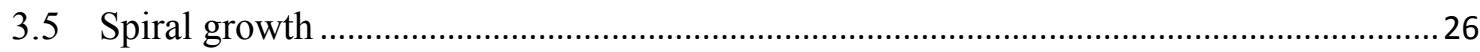

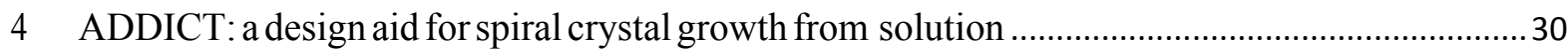

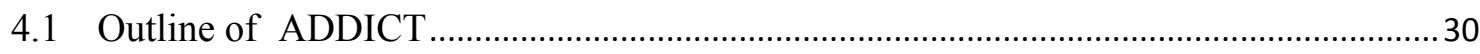

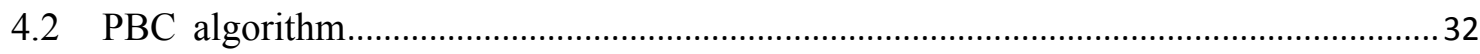

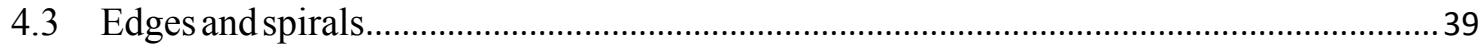

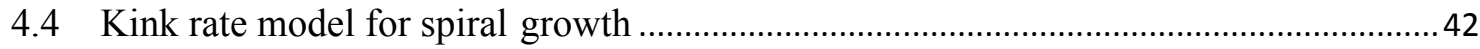

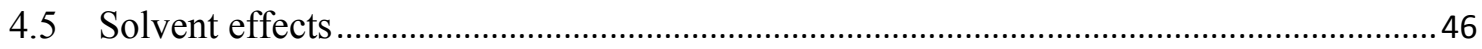

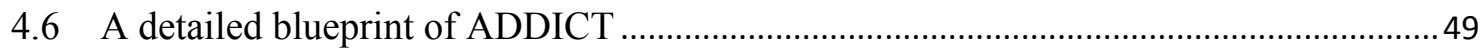

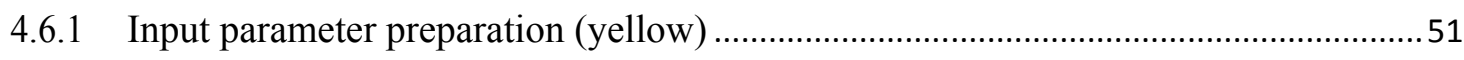

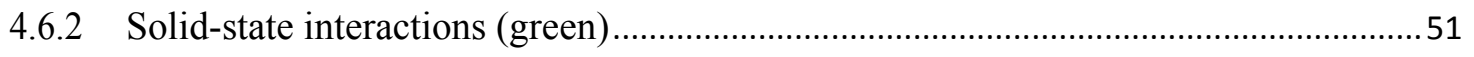


4.6.3 Plane and PBC determination (orange) .....

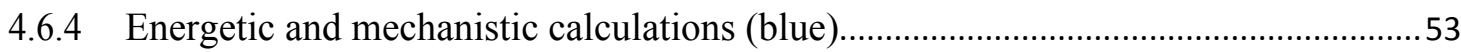

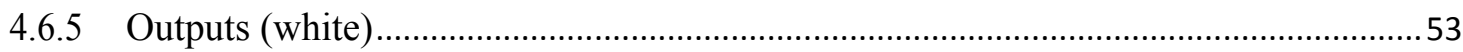

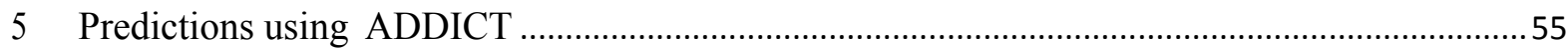

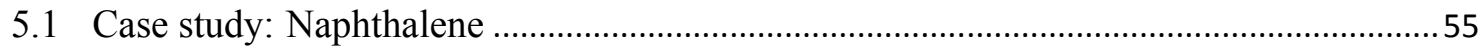

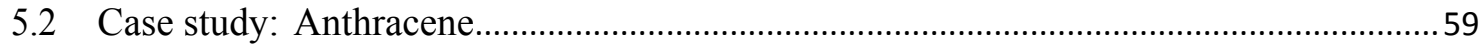

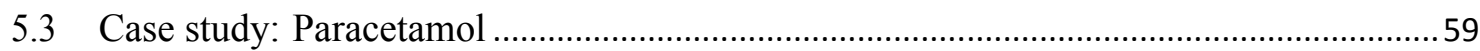

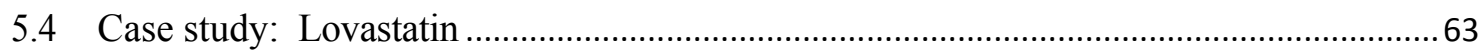

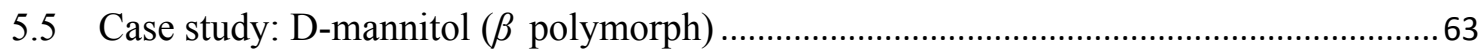

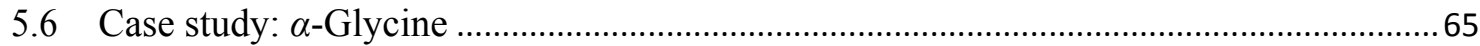

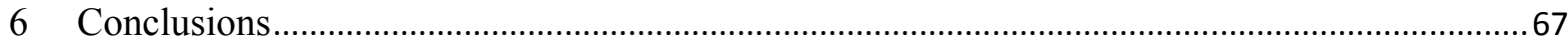

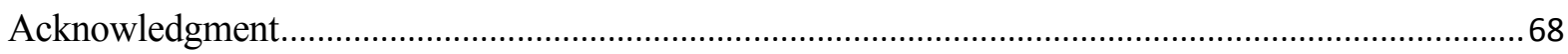

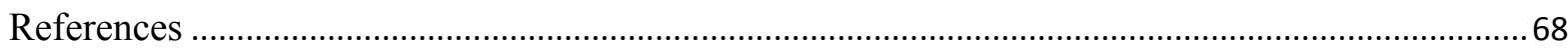




\section{Introduction}

Crystal morphology plays an important role in pharmaceutical manufacture and other practical applications, with different crystal habits (i.e. rods, needles, plates, blocks) possessing different physicochemical characteristics [1-4]. Bioavailability is central to pharmaceutical efficacy and can be impacted by the crystal shape [4-6]. Additionally, highaspect-ratio, needle-shaped crystals are typically undesirable for pharmaceutical applications, where the shape impacts downstream processing such as filtration [7-8], but can be preferred for the active layer morphology to enhance performance in electronic applications [9-10]. Figure 1.1 demonstrates a variety of crystal habits for pharmaceutical crystals; their faceted nature is evident with well-defined crystallographic planes. Control over the environmental conditions during crystal growth, such as solvent, temperature, supersaturation and the presence of impurities/additives/stabilizers enables different morphologies to be accessed; consequently subsequent processing and end-use functionality are strongly affected. Often an optimum or at least improved morphology can be imagined based on knowledge of the product's function; considering a catalyst for example, the ideal shape would be dominated by the most catalytically active faces [3].

With an improved understanding of how crystal shape affects product functionality, the ability to synthesize a particular morphology will become even more desirable. Scientifically and systematically engineering the shape of crystalline solids remains a challenge, however, with the large design space for growth conditions typically being navigated experimentally $[1,12]$; this paves the way for modeling to support crystal engineering. A useful model should have high chemical fidelity so as to capture effects such as temperature, solvent and supersaturation from a mechanistic basis using the underlying growth physics. We have developed such a theoretical 
framework for predicting the crystal habit in the form of proof-of-concept, academic software called ADDICT (Advanced Design and Development of Industrial Crystallization Technology) that aims to provide a pathway to predictive modeling and crystalline product design. Effectively, our model considers established theories of crystal growth and incorporates these into a framework that predicts the crystal shape using the underlying chemistry rather than the oftenused general trends that fail to reproduce observed morphologies.

(a) Rods

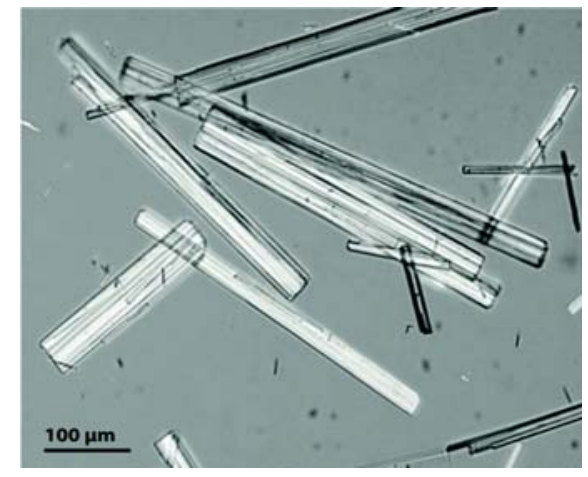

(c) Needles

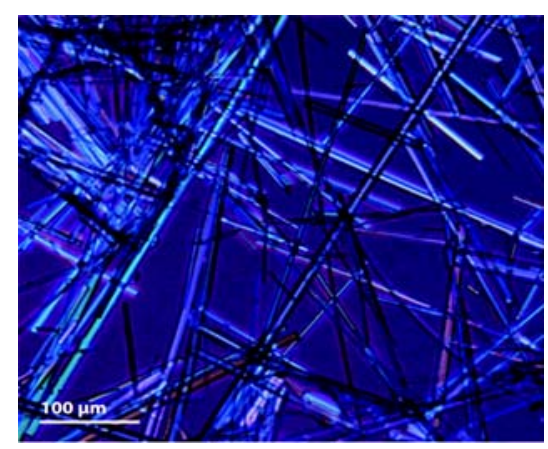

(b) Blocks

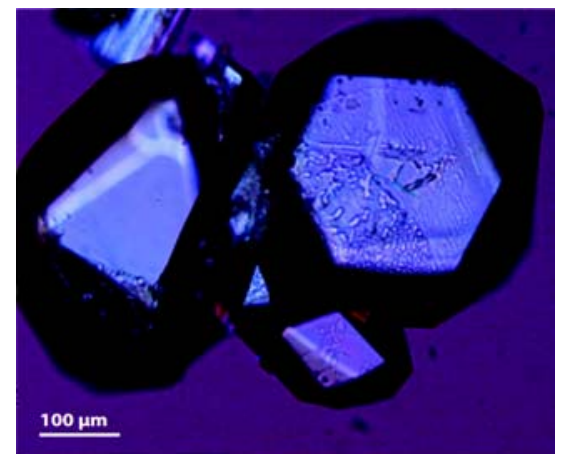

(d) Plates

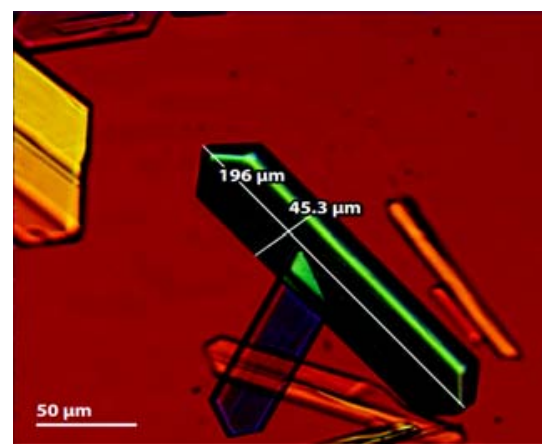

Figure 1.1: Examples of crystal growth shapes under optical microscopy: rods (a), blocks (b), needles (c), plates (d). Image courtesy of N. Variankaval, Merck and Co. (private communication) [11]. 
ADDICT aims to be a visual and convenient prototype tool useful for both theorists and experimentalists that is simple enough for novice users to obtain rapid predictions while enabling more experienced users extra functionality to cater for advanced design. ADDICT is based on a recent model developed [13] for organic non-centrosymmetric solute molecules growing under the spiral growth mechanism proposed by Burton, Cabrera and Frank [14]. This model accounts for the non-isotropic behavior of complex organic molecules and performs well at predicting the growth habit of pharmaceutical molecules; it has recently been adopted outside of our group for various crystalline explosives, with successful results also [15-17]. Central to the model are calculations of the net rates of solute attachment into kink sites on spiral edges, from which the normal growth rate of each face results.

This article reviews crystal growth models presented in the literature and provides a detailed overview of ADDICT's structure and how mechanistic theories for a faceted crystal have been incorporated in an automated fashion. The resulting blueprint describes the process of obtaining a crystal growth shape prediction for a real (i.e., complex, non-centrosymmetric) system, including the effect of solution environment. ADDICT is poised to become a nextgeneration tool to predict crystallization of active pharmaceutical ingredients (APIs) or other organic molecular crystals that are often manufactured under carefully controlled spiral growth conditions. The main development of ADDICT has been to organize and automate an existing growth model, so that few inputs are required: an experimental crystallography determination must be provided (e.g., CIF file), a Gaussian electron density calculation performed (to provide partial charges on the atoms of a growth unit), and growth conditions provided (e.g., temperature, solvent and supersaturation); quick morphology predictions are then obtained (on the order of seconds or minutes for a standard desktop PC). ADDICT is currently optimized for small organic molecules, though future modifications are planned to 
broaden the applicability to organic salts, via inclusion of recent developments for ionic systems [18-25].

\section{Non-mechanistic models for crystal habit}

Gibbs developed the first criteria for describing equilibrium crystal shapes [26], with the underlying principal that the total surface free energy of the fluid-solid interface should be minimized. The work of crystallization can be expressed by the Gibbs-Thomson formula [27]:

$$
\Delta G=-\frac{V \Delta \mu}{V_{M}}+\sum_{i} \gamma_{i} A_{i}
$$

where the reward for crystallization from a supersaturated solution is based on the chemical potential difference $\Delta \mu$ between a growth unit (e.g., molecule, dimer, ion, cluster, etc.) in the growth environment and in the crystal $\left(V\right.$ and $V_{M}$ are crystal and growth unit volumes, respectively); the penalty is due to surface energies $\left(\gamma_{i}\right)$ of each facet (having area $A_{i}$ ). The equilibrium shape corresponds to the minimum in $\Delta G$, which for a fixed crystal size implies:

$$
\sum_{i} \gamma_{i} d A_{i}=0
$$

i.e., the surface free energy is minimized. The shape that solves this problem is given by the famous Wulff construction [12, 27-30]:

$$
\frac{\gamma_{1}}{H_{1}}=\frac{\gamma_{2}}{H_{2}}=\cdots=\frac{\gamma_{i}}{H_{i}}
$$

$H_{i}$ is the perpendicular distance of face $i$ from the crystal center, which is, therefore, proportional to its surface energy for the equilibrium shape. This shape ensures that the lowest energy faces are dominantly expressed on the morphology and high surface energy faces can 
be excluded if their $H_{i}$ is large enough to produce a plane geometrically beyond where adjacent faces would intersect (i.e., high energy faces lie outside the convex hull). Note that the orientation of each face is fixed based on the crystallography, which determines the angles between planes in forming this construction and also gives rise to the anisotropic $\gamma_{i}$ 's based on the distinct crystallographic surface structures.

In contrast to fluids, however, solids offer a strong resistance to shape deformation; essentially, there is an activation energy that typically prevents an equilibrium shape being achieved. The barrier to reorganization is reduced with size, leaving nanocrystals as potential candidates to reach their equilibrium shape, but for most crystalline products it is kinetics, instead of thermodynamics alone, that is expected to govern the crystal morphology. This observation was remarkably noted by Gibbs as a footnote $[12,26]$, stressing that the kinetically slow-growing faces determine the crystal habit and $\sum_{i} \gamma_{i} A_{i}$ will not usually be minimized.

One can predict the actual (non-equilibrium) crystal habit providing the perpendicular growth rate of each face, $G_{i}$, is known. For constant $G_{i}$ (i.e., in an unchanging growth environment), there exists a steady-state morphology that a crystal will evolve towards regardless of its initial seed shape [31,32]. This can be calculated using the Frank-Chernov condition $[33,34]$ :

$$
\frac{G_{1}}{H_{1}}=\frac{G_{2}}{H_{2}}=\cdots=\frac{G_{i}}{H_{i}}
$$

where $\mathrm{H}_{\mathrm{i}}$ is again the perpendicular distance of face $\mathrm{i}$ from the crystal center. This is analogous to the Wulff construction with surface energies replaced by growth rates. Therefore, the path to predicting real crystal shapes lies in determining the perpendicular growth rate $\mathrm{G}_{\mathrm{i}}$ of each crystallographic face, which via equation 4 fixes the relative $H_{i}$ 
distances and provides the shape.

\subsection{BFDH model}

The initial foray into a non-equilibrium model for crystal growth morphology was provided by Bravais, Friedel, Donnay and Harker [35-37] via the now-termed BFDH model. This was the first approach at predicting crystal growth rates, which through the Frank-Chernov condition (equation 4) allows one to predict the steady-state crystal morphology. The BFDH model takes the form of a trend between growth rate and crystallographic structure; the perpendicular growth rate $G_{h k l}$ is assumed to be inversely proportional to the interplanar spacing, $d_{h k l}$, between successive planes with Miller indices $(h k l)$ :

$$
G_{h k l} \propto \frac{1}{d_{h k l}}
$$

This method is solely based on geometrical crystallographic considerations so does not account for any solid-state energetics or modifications from the growth environment. This fixed interplanar spacing for each $(h k l)$ plane can be calculated using the following equations [38]:

$$
\begin{gathered}
d_{h k l}=\sqrt{\frac{1}{r_{h k l}}} \\
r_{h k l}=\left(1-\cos ^{2} \alpha-\cos ^{2} \beta-\cos ^{2} \gamma+2 \cos \alpha \cos \beta \cos \gamma\right)^{-1}\left[\frac{h^{2}}{a^{2}} \sin ^{2} \alpha+\frac{k^{2}}{b^{2}} \sin ^{2} \beta+\frac{l^{2}}{c^{2}} \sin ^{2} \gamma\right. \\
\left.+\frac{2 k l}{b c}(\cos \beta \cos \gamma-\cos \alpha)+\frac{2 l h}{a c}(\cos \alpha \cos \gamma-\cos \beta)+\frac{2 h k}{a b}(\cos \alpha \cos \beta-\cos \gamma)\right]
\end{gathered}
$$

where $h, k, l$ are Miller indices, $a, b, c$ are unit cell dimensions and $\alpha, \beta, \gamma$ are the corresponding crystallographic angles. X-ray extinction (symmetry) conditions for the space group should also be considered to select the appropriate Miller indices in each direction, 
upon which the growth rates are fixed for a crystal system and the morphology can be determined.

The BFDH model is easily implemented since it merely requires knowledge of the crystallography. It is commonly used for this advantage and is built into the commercial software Mercury [39] from the CCDC. While the general trend that faces with large interplanar spacings dominate the crystal habit can be true (best results are obtained for the case of vapor growth [40-41]), using equation 5 to make a quantitative prediction of crystal shape usually fails to match experimentally observed morphologies, particularly for solutiongrown crystals [41-42]. For a given crystallography (i.e., fixed $a, b, c, \alpha, \beta \& \gamma$ ) it is the Miler indices that determine $d_{h k l}$ via equations 6-7; high-Miller-index faces have a small $d_{h k l}$ and are usually not displayed on the steady-state crystal habit due to their commonly high growth rates (a trend that equation 5 does predict). A crystal system has only a single shape prediction via the BFDH model (defined by the crystallography) regardless of growth environment, rendering the BFDH model additionally powerless to predict morphological changes as a result of solvent, supersaturation, additives or other imposed growth conditions. Its use should, therefore, be limited to a qualitative prediction of the faces that are likely present for a given crystal; actual shape predictions are ill-advised since the model has no consideration of energetics or surface chemistry effects.

\subsection{Hartman-Perdok theory}

In 1955, Hartman and Perdok published three landmark papers [43-45] in the science of crystal growth. They established a connection between solid-state interactions and crystal morphology, proposing the concept of periodic bond chains (PBCs) and their use to classify crystal faces. 
A periodic bond chain is a repeating array of growth units in a single direction held together by strong inter-growth-unit interactions within the crystal. For organic molecular crystals, these strong interactions usually represent the short bonds within the first coordination sphere around a particular growth unit in the lattice [43-46]. As a result of these favorable interactions, on each face crystalline surface structures are typically bounded by the in-plane PBCs (a PBC vector denotes the full translation distance of the chain's repeat unit in the PBC direction). These strong interactions represent bonds formed between growth units during crystallization [43-46] and therefore exclude any intra-growth unit interactions. More detailed properties of PBCs, including our algorithm for their determination in complex systems and additional rules for ionic crystals, are presented in section 4.2.

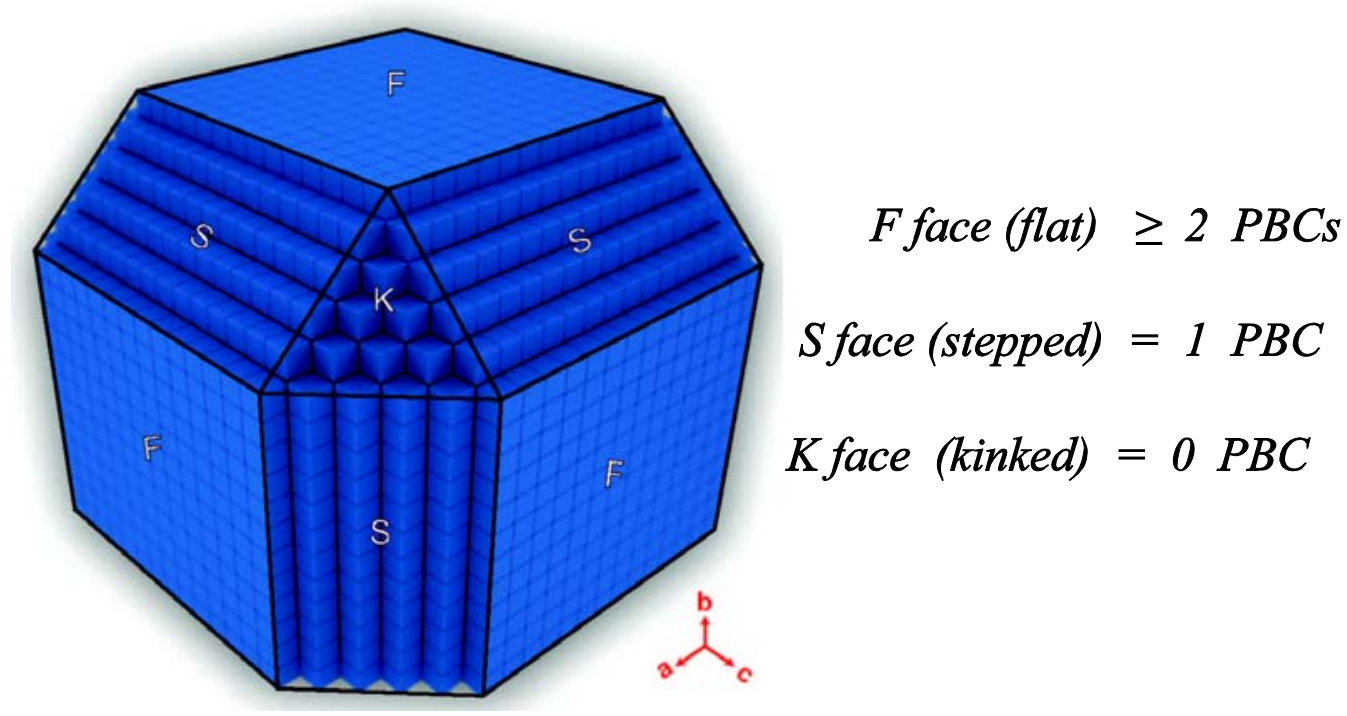

Figure 2.1: F, S and K face classification for a Kossel crystal (cubic growth unit with isotropic interactions). Image adapted and reprinted from Lovette et al. [12] with permission from ACS

Hartman and Perdok also introduced a qualitative theory of crystal faces according to their in-plane PBCs, where faces fall into three categories: F (Flat), S (Stepped) and K 
(Kinked), as shown in Figure 2.1. The dominant faces on the steady-state crystal morphology are slow growing and almost always F faces, which contain two or more PBCs and are, therefore, flat and stable by way of the in-plane growth unit interactions. S faces, containing only one $\mathrm{PBC}$, and $\mathrm{K}$ faces, containing no $\mathrm{PBCs}$, have high surface energies (from broken PBCs extending out-of-plane) and usually grow extremely fast.

\subsection{Attachment energy (AE) model}

Following the connection between solid-state interactions and characterization of faces, Hartman and Bennema developed [47] the attachment energy (AE) model as a simple method that could predict crystal growth rates relatively well in lieu of a fully mechanistic model. Since its inception this model has been used extensively to predict crystal shapes [41,48-52] and remains routinely used today. The underlying principle, which can be proved, is that if stronger bonds are formed when a growth unit attaches to a face, then this process will require less time and lead to a faster growth rate $[43,47,53]$ :

$$
E_{m}^{\text {att }}>E_{n}^{a t t} \Rightarrow G_{m}>G_{n}
$$

with $m$ and $n$ being two different faces. The attachment energy for a face $(h k l), E_{h k l}^{a t t}$, is defined as the energy per growth unit released upon crystallization of a new slice of thickness $d_{h k l}$, thus considering interactions perpendicular to the terrace alone (equivalently, it represents the energy to remove a terrace-adsorbed growth unit) [43]. For each plane, the lattice energy (which is constant for the crystal, representing the total lattice interaction energy per growth unit) is divided between this attachment energy (out-of-plane interactions) and the slice energy (in-plane interactions for slice $\left.d_{h k l}\right)[47,53]$ :

$$
E^{\text {latt }}=E_{h k l}^{\text {att }}+E_{h k l}^{\text {slice }}
$$


where each energy is usually reported as positive.

While faces with a larger attachment energy have faster growth rates, without a functional form for the relationship between $G_{h k l}$ and $E_{h k l}^{a t t}$, no quantitative shape predictions can be made. When using the AE model, therefore, growth rates are typically assumed proportional to attachment energies [54]:

$$
G_{h k l} \propto E_{h k l}^{a t t}
$$

This assumption has no physical basis and was introduced purely to enable shape predictions by way of the Frank-Chernov condition once attachment energies for each face have been calculated. Even for simple crystal systems containing high symmetry, mechanistic expressions remain non-linear and do not collapse to equation 10. Nonetheless, the approximation can occasionally hold and the AE model usually predicts the correct morphological faces (since low attachment energies lead to low perpendicular growth rates and faces that dominate the morphology).

The AE model is generally an improvement on the BFDH model due to the inclusion of solid-state energetics. Although these interactions, albeit on a more complicated level, do govern the physics of crystal growth, the functional form for the AE model remains arbitrary. As such, the AE model should not be expected to perform well at predicting the crystal shape universally or for complex systems and in particular, the influence of external factors on crystal growth, such as solvent, additives, and supersaturation are not included.

\subsection{Modified attachment energy (MAE) models}

There is growing interest in using modified attachment energy (MAE) models [55-61] to capture effects of the growth environment, such as solvent, supersaturation or additives, which the AE model has no architecture for dealing with. Under MAE approaches, molecular 
simulations are typically used to model how the growth environment interacts with a crystal face, introducing solvent and/or solute molecules around relevant surface sites and determining resulting energetics (typically binding energies, which are used with $E_{h k l}^{\text {att }}$ to create a composite MAE variable). Adopting molecular simulations to study the interface can provide a much more sophisticated picture of the surface chemistry than what is currently able to be incorporated into rapid mechanistic methods (see Section 4.5) and much insight can be gained by considering the crystallization process in such detail. Nonetheless, the drawback to using MAE models for shape predictions, beyond the computational expense, is the need to assume or correlate a functional form for the MAE variable and its relation to face growth rates. Developed MAE models may produce agreement with observed morphologies in certain cases, but with no grounding in mechanistic theories their ability to predict the crystal habit of a general system appears limited.

\section{A mechanistic treatment of crystal growth}

Neither the BFDH nor AE model is able to provide a high-fidelity description of crystal morphology as a function of the growth environment. Instead, to describe real crystal morphologies a model must consider the anisotropic growth rates of the crystallographic faces, as Gibbs himself emphasized. One class of methods that can achieve high-fidelity morphological predictions is Monte Carlo simulations. A noteworthy example of such an approach is the MONTY algorithm [62-63], which considers the influence of supersaturation, temperature, concentration, and dissolution free energy. The chief disadvantage of using such methods is their investment both computationally and in terms of required expertise, which unfortunately renders them unsuitable for rapid predictions under different growth conditions for a variety of crystal systems. Unfortunately, speed and ease of use are both critical for a 
modeling technique to gain traction industrially as a screening step. To combat this need, ADDICT has instead been developed based on mechanistic models. It should nonetheless be noted that KMC simulations can provide extremely valuable insight to the mechanistic approaches contained herein, having been developed concurrently to account for the same underlying physics of crystal growth. Additionally, the demonstrated ability to account for supersaturation shape dependence as a result of different growth mechanisms [17], for example, remains beyond the currently capability of ADDICT. The mechanistic theories contained within ADDICT will now be presented.

Stable incorporation of growth units into the crystal occurs where multiple bonds can be formed with neighboring growth units in the lattice. Stepped and (particularly) kinked faces are typically those planes with higher Miller indices and have an extremely high density of these stable incorporation sites. This usually leads to a fast rough growth mechanism (section 3.3) and these faces do not commonly appear on steady-state morphologies; if they are present it will result in a high-aspect-ratio needle or platelet shape [12]. The crystal habit is instead bounded by the slower-growing F faces, which typically grow under a layer-by-layer mechanism. Layered growth is amenable to modeling, consisting of the forward motion of step edges across a face to complete successive crystal layers [14]. A forward step velocity is achieved through a positive net rate of growth unit attachment to the step edge and the most stable positions for attachment are at kink sites [11,12,14,64-68]. Attachment at and detachment from kink sites are the competing kinetic processes that influence the step velocity on each edge on each face [69], which in turn influences growth rates and the crystal shape. Surface physics and chemistry (including the growth environment) define the rates of these processes. 


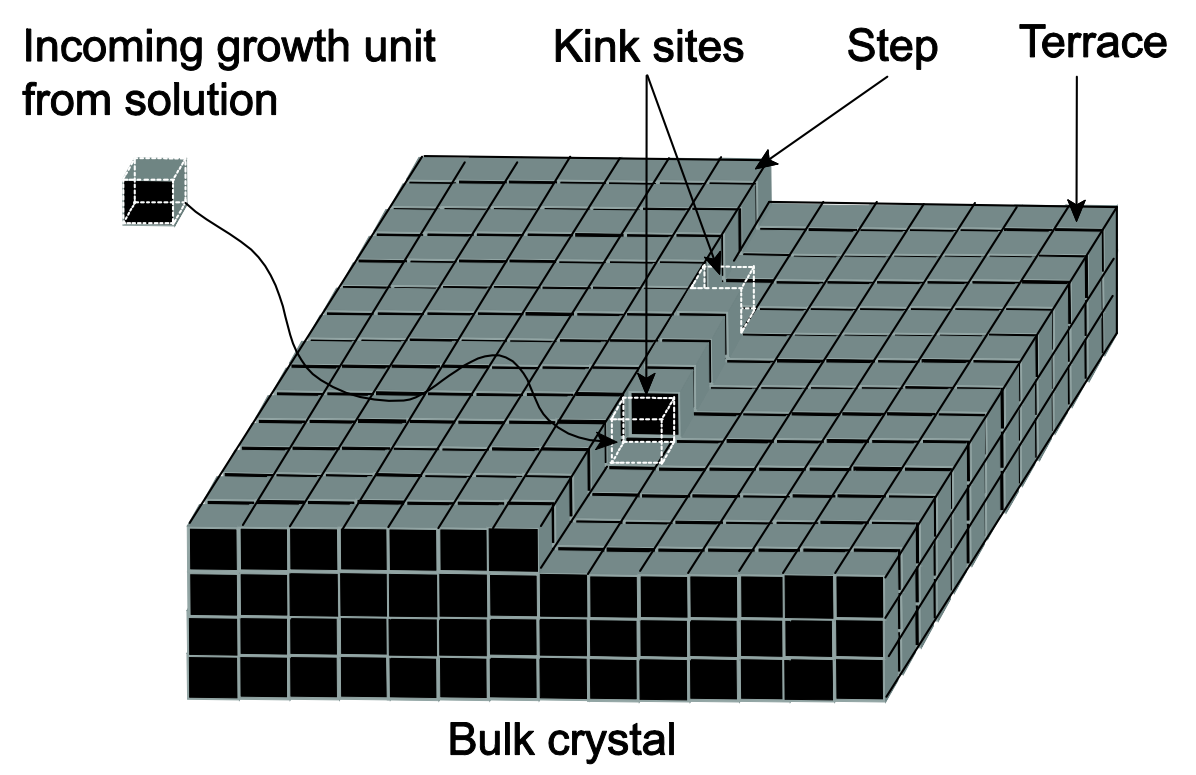

Figure 3.1: Schematic illustration of the surface structure on a faceted crystal with cubic growth units, indicating kink sites, step edge and the terrace. The incorporation mechanism is, in principle, system-specific but under solution growth for organic molecular crystals typically involves sequential adsorption at the terrace and then the step before entering the kink site [11,14,72-76].

Figure 3.1 shows an example surface structure of a step edge on an F face, with kink sites indicated. As can be seen, the forward propagation of a step edge acts to complete the incomplete face layer. Kink sites are renewable upon attachment and furthermore are continually regenerated via thermal roughening [12,14,70,71] (see Figure 3.2 (a) \& (b)). Centrosymmetric crystals necessarily have the same kink-site interactions on each edge and face, but for non-centrosymmetric crystals each edge on each face can generally have multiple distinct kink sites containing different sets of interactions. Layered growth mechanisms often give rise to a tiered array of steps moving across the surface (Figure 3.2 (c)), completing several crystal layers simultaneously that leads to the normal growth rate of the face. 
Steps are generated on $\mathrm{F}$ faces as a result of two mechanisms: two-dimensional (2D) nucleation on the surface or screw dislocations (leading to spiral growth); these are discussed in sections section $3.4 \& 3.5$, respectively. Since both mechanisms are able to operate concurrently, whichever leads to the fastest normal growth rate will dominate on a face. The dominant mechanism can be different for different faces on the same crystal and depends on both growth conditions and surface interactions (both solid-state and interfacial). At low supersaturation, the spiral growth mechanism with steps emanating from a screw dislocation is dominant [14]. As the supersaturation increases, 2D nuclei form more readily and a $2 \mathrm{D}$ nucleation $\&$ growth regime can overtake the spiral mechanism. At a sufficiently high supersaturation an $\mathrm{F}$ face can also transition to a rough growth regime $[12,77]$; these regimes are illustrated in Figure 3.3 and the progression between regimes is shown in Figure 3.4. Spiral and 2D nucleation \& growth mechanisms can be readily identified upon imaging the surface structure of grown crystals [78,79] and transitions between regimes have also been observed experimentally for various systems, notably through the use of in-situ atomic force microscopy (AFM) [80,81]. 

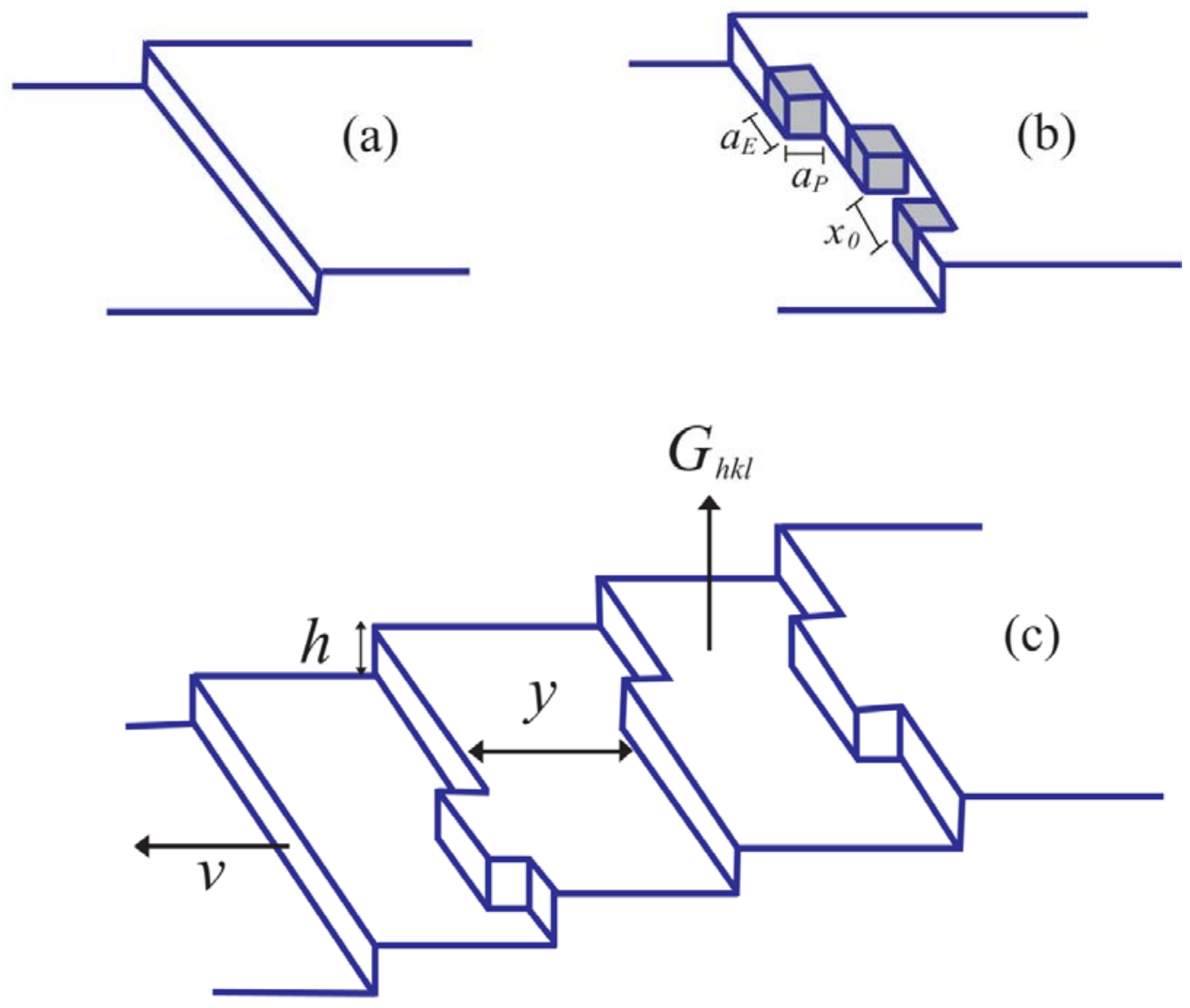

Figure 3.2: At $0 K$ the step edge is straight (a), while above $0 K$ the propensity for rearrangement via thermal fluctuation leads kinks to exist at all times (b) $[12,14,70,71]$. The propagation length for addition of a row of growth units is $a_{P}$, the width of a growth unit along the edge is $a_{E}$ and the number of sites between kinks is termed $x_{0}$. (c): The forward velocity ( $v$ ) of successive steps gives rise to the normal growth rate of the face, $G_{h k l}$; $h$ is the step height (usually the interplanar spacing $d_{h k l}$ ) and $y$ is the inter-step distance (regular under spiral growth - see section 3.5). Image adapted and reprinted from Lovette et al. [12] with permission from ACS. 
The driving force for crystallization is a chemical potential difference between the solute in its growth environment and in the crystalline lattice. This chemical potential difference can be defined in terms of the supersaturation S $[67,82]$ :

$$
\Delta \mu=k_{B} T \ln S
$$

This supersaturation can be defined as $S=C / C_{\text {sat }}$ for solution growth and $S=P / P_{\text {sat }}$ for vapor growth. For $\mathrm{S}=1$ the environment is saturated and crystals do not change in size, while growth occurs in the supersaturated state $(S>1)$ and dissolution occurs in the under-saturated state $(S$ $<1$ ). Note that equation 11 does not require the solution to be ideal; it assumes only that the activity coefficients of a saturated solution and a supersaturated solution are approximately equal. Characteristics of vapor and solution growth environments are listed below.

\subsection{Vapor (sublimation) growth}

In vapor growth, solute particles are provided from a source via sublimation and are deposited directly into the growing crystal. A temperature difference between source $\left(T_{S}\right)$ and the growing crystal $\left(T_{D}\right)$ is usually used to drive crystallization, which the supersaturation is often

expressed as; $S=T_{S} / T_{D}$ should not simply be substituted into equation 11 , however. Features of the vapor growth include the following:

(a) Growth rates can be slow with the small amount of solute vapor impinging on the crystal. This can be useful for facile surface manipulation (e.g., semiconductor manufacture), but is typically inefficient for growing larger crystals [83]. 


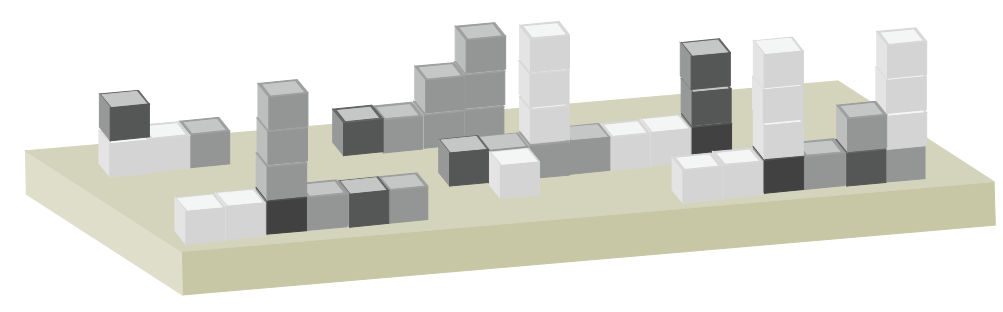

(a) Rough growth
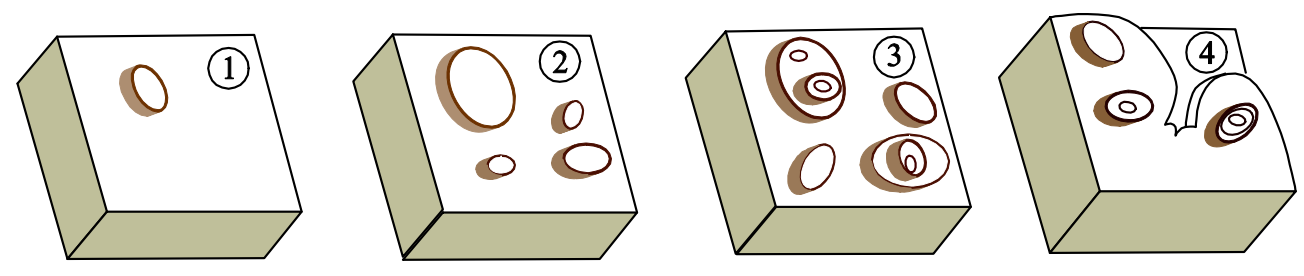

(b) 2D nucleation \& growth
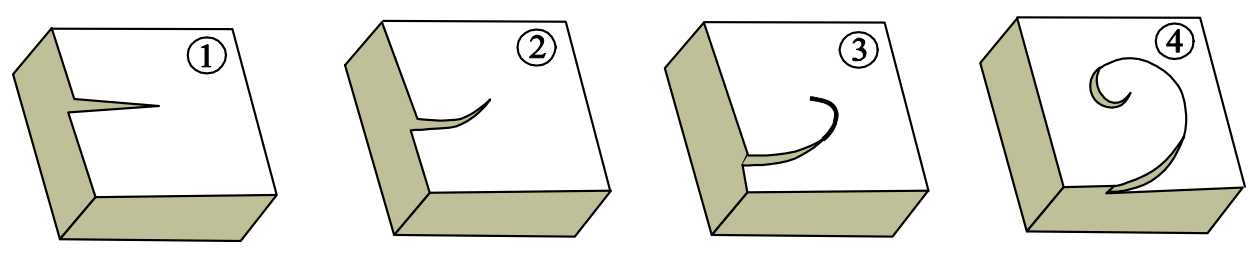

(c) Spiral growth via screw dislocaton

Figure 3.3: Illustrations of various growth mechanisms. Rough growth (a) can occur at high supersaturation on an $\mathrm{F}$ face when attachment is favorable at all surface sites. In layered growth, continual 2D nucleation and the collective growth of resulting nuclei can complete various layers (b), or a screw dislocation can provide a continuous source of steps in the form of a growth spiral (c). Note that 2D nuclei and growth spirals are normally faceted. 


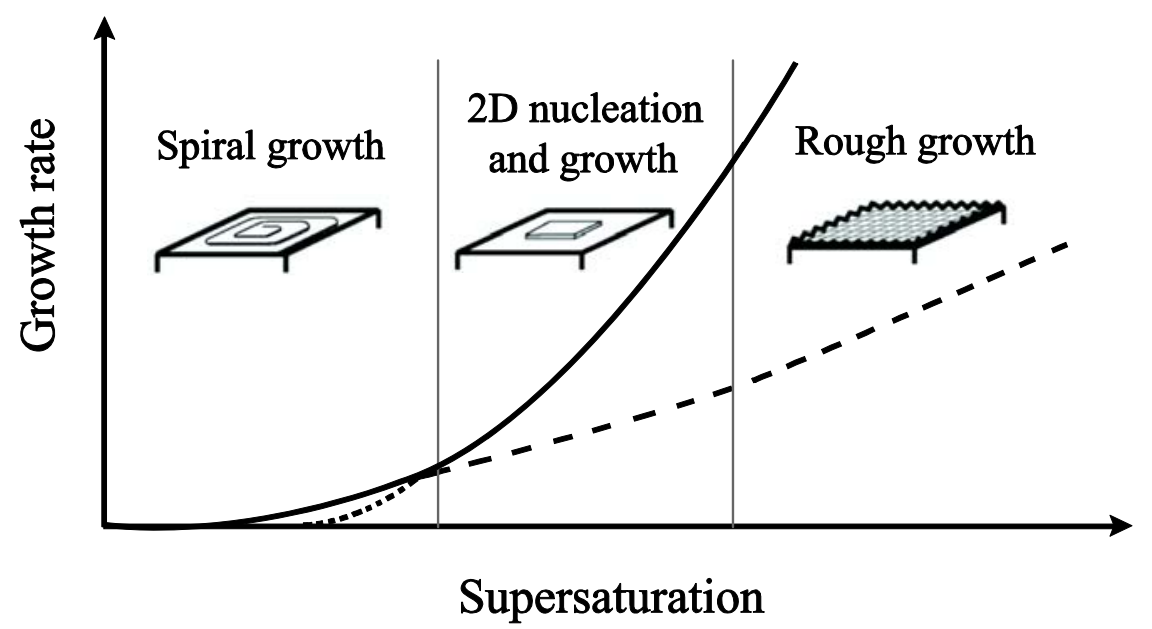

Figure 3.4: A typical progression of growth mechanisms on an F face is illustrated. At low supersaturation the spiral growth mechanism (solid line) is faster than 2D nucleation $\&$ growth (dotted line) and is the dominantly observed mechanism. Above a certain supersaturation, however, the 2D nucleation $\&$ growth rate (now drawn as a solid line) can overtake the spiral mechanism (the dashed line represents a continuation of the spiral growth rate expression). Eventually a rough growth regime may be reached. Image reprinted from Lovette et al. [12] with permission from ACS.

(b) Well-defined facets often form to define the crystal [83]. This can be reasoned through the relatively high surface energies in the absence of favorable solvation effects, which creates a large driving force to crystallize in the most favorable surface structures. Note that this effect is still felt mechanistically even when the Wulff shape is not achieved.

(c) The barrier to attachment is low, consisting primarily of the need to properly orient into the lattice position [82]. Surface diffusion effects are, therefore, expected to be more important than in solution growth, where relatively higher attachment barriers (representing the required desolvation - see below) usually result in a kinetic surface-integration-limited regime. 


\subsection{Solution growth}

The chemical potential driving force in solution is a result of the excess concentration of solute in solution above the saturated concentration. Both the crystal surface and solute growth units in solution are solvated, so desolvation must occur upon approach of a growth unit to the surface in order for attachment to occur. This required desolvation represents the principal energetic barrier in solution growth [67,69], which can be significant and often leads to a kinetic regime where the attachment process determines the rate of crystal growth. The various processes taking a growth unit from solution to being attached at the kink site are listed below (see also Figure 3.1) [11, 14, 72-76]:

(a) Bulk transport (convection/diffusion) from solution to the terrace.

(b) Terrace adsorption (partial desolvation).

(c) Surface diffusion across the terrace to a step edge.

(d) Edge adsorption (partial desolvation).

(e) Diffusion along the edge to a kink site.

(f) Attachment to a kink site (remaining desolvation).

This picture is for an organic molecule; in contrast, inorganic growth units are expected to attach at kink sites directly from solution due to high diffusive barriers on the surface resulting from like-charge repulsion. Coincident with desolvation, the latent heat of crystallization is released and must be transported away, though this is expected to occur on a much faster timescale than the processes described above [73].

The growth rate of a crystal face is limited by the slowest of these processes $[73,84]$. For molecular organic crystals grown from solution in the spiral growth regime, kink attachment (i.e., surface integration) is expected to be rate-limiting [67]. The density of kink sites on the step is, therefore, an important parameter influencing the growth rate and depends on the 
strength of intermolecular interactions (discussed later).

\subsection{Rough growth}

In contrast to layered growth, rough growth occurs when favorable attachment is available across the entire crystal face. This is by definition the case on stepped (S) and particularly kinked $(\mathrm{K})$ faces, which grow under a rough mechanism from any environment. The growth rate of S \& K faces are usually significantly higher than $\mathrm{F}$ faces due to this increased density of kink sites [12]. Rough growth can also occur on $\mathrm{F}$ faces if the environment (e.g., supersaturation, temperature, etc.) is such that attachment is favorable at terrace and step sites in addition to kinks, i.e., a roughening transition has been reached which will destroy any surface structures from layered mechanisms [12,77].

Under the rough mechanism, crystal growth is expected to be limited by transport of solute growth units to the surface, instead of attachment kinetics. This bulk transport- limited growth rate, $G_{B T}$, is then proportional to the concentration driving force between bulk solution $(C)$ and the crystal surface ( $C_{\text {sat }}$ under fast surface integration) [12,82]:

$$
G_{B T} \propto\left(C-C_{s a t}\right)
$$

Crystals under rough growth tend to lose their faceted nature and are instead bounded by high index planes or rounded, non-crystallographic surfaces. This leads to spherulitic shapes, or, in extreme cases of transport-limited growth, fractal and dendritic morphologies [85].

\subsection{Two-dimensional (2D) \& nucleation growth}

The 2D nucleation \& growth mechanism was the first model used to describe layered crystal growth $[64,65,86]$. With increasing supersaturation, the 2D nucleation rate increases and the critical size of a 2D nucleus decreases [87], so in the limit of high supersaturation this mechanism essentially represents a rough regime. The critical size of a $2 \mathrm{D}$ nucleus can be 
determined via classical nucleation theory: it is the size beyond which growth is favorable over dissolution [87], which represents a maximum in free energy when considering the volume reward of crystallization (due to the chemical potential difference $\Delta \mu$ ) against the surface energy penalty.

At low nucleation rates a single $2 \mathrm{D}$ nucleus can grow to the face edges and complete the layer before an additional nucleation event (mononuclear growth), while at higher nucleation rates (e.g., higher supersaturation) multiple nuclei form and collectively complete face layers following their subsequent growth across the surface (termed a birth and spread regime) $[12,68,82,83,87]$. The growth rate under $2 \mathrm{D}$ nucleation depends, therefore, on the coverage time.

Earlier models for 2D nucleation rely on simplifying assumptions such as circular nuclei, constant spreading velocities or Kossel growth units [88,89]. Notable recent models exist for the birth and spread regime, considering anisotropic spreading for rectangular [90] and general nucleus shapes [87]. From a simulation perspective, Monte Carlo algorithms [17,62,63,91-96] and Molecular Dynamics [95,97-99] have each been utilized in modeling 2D nucleation (and also the spiral mechanism below), providing valuable insight to this growth mechanism. In order to reduce complexity these techniques have often studied simplified systems such as sublimation growth, Kossel crystals or a single supersaturation. In terms of providing rapid shape predictions for screening purposes, however, these approaches remain too slow due to the computational expense and the fact that growth mechanisms for each face are common inputs that may have to be determined for each set of tested growth conditions before use. 


\subsection{Spiral growth}

The spiral growth mechanism reconciles the discrepancy between observed crystallization at low supersaturation and the far lower growth rates expected for $2 \mathrm{D}$ nucleation \& growth within such an environment. This spiral mechanism was developed by Burton, Cabrera and Frank [14] (BCF) in arguably the most revolutionary paper within the field of crystal growth. Noting that kink sites on steps were critical for growth unit incorporation, they proposed that a screw dislocation could provide a continuous source of steps for a self-sustaining growth mechanism rather than waiting for $2 \mathrm{D}$ nucleation events that would become increasingly scarce at low supersaturations. This explains the dominance of spirals at low supersaturation (Figure 3.4) and accounting for this mechanism is critical to describing the growth of crystals under such conditions. The controlled crystallization of APIs often uses a low supersaturation to ensure good product purity and a uniform, faceted crystal morphology; for this reason ADDICT adopts a mechanistic model for spiral growth to predict the crystal morphology.

Faces of a real crystal are rarely perfect and so screw dislocations exist almost universally, enabling the spiral mechanism to operate for most systems. As growth units attach to the exposed step at the dislocation and lead to its advance across the surface, an adjacent edge appears. There is usually a critical length before this newly exposed edge itself advances, at which point the next adjacent edge appears and the process repeats [100]. Therefore, the emerging edges essentially rotate about the point of the screw dislocation and eventually the original edge reappears one layer higher (corresponding to the interplanar spacing, $d_{h k l}$, for an elementary step height). Each edge continues its advance outwards to the face's ends (or upon meeting another step or equivalent boundary), and so the action of the rotating spiral leads to a normal growth rate of the face, with steps continually emerging from the spiral in the edge directions, separated by a regular distance called the inter-step spacing. 
This process is illustrated in Figure 3.5.

For the BCF spiral mechanism, the growth rate of face $(h k l), G_{S, h k l}$, has been expressed as $[12,100]:$

$$
G_{S, h k l}=\frac{h v}{y}=\frac{h}{\tau_{S}}
$$

where $h$ is the step height (e.g., $d_{h k l}$ or a simple multiple of it), $v$ is the step velocity and $y$ is the inter-step distance $(y / v$ represents the time taken to advance the face a single layer of growth units in the normal direction - see also Figure 3.2 (c)). This time $y / v$ is additionally equal to the spiral rotation time, $\tau_{S}$. With the step height defined for each face based on the crystallographic geometry, the problem of calculating a face's growth rate under the spiral growth mechanism is essentially reduced to finding $\tau_{s}$.

To determine $\tau_{S}$, one must consider both the critical length and the step velocity of each spiral side. For kinetically limited growth, which is expected at low supersaturations where the spiral regime operates, one must consider both the density of kink sites on a step as well as the net rate of solute incorporation into each kink site [13]. This net attachment rate is termed the kink rate, $u_{i}$, and is the balance between attachment and detachment events that can be considered as elementary reactions $[66,101]$. 


$$
\text { Top view Perspective view }
$$

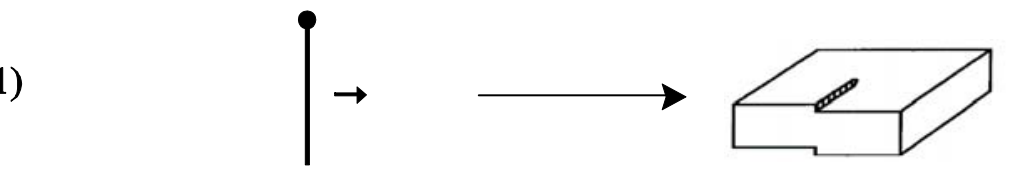

(2)

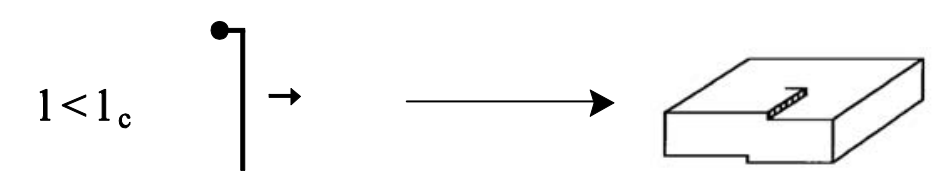

(3)

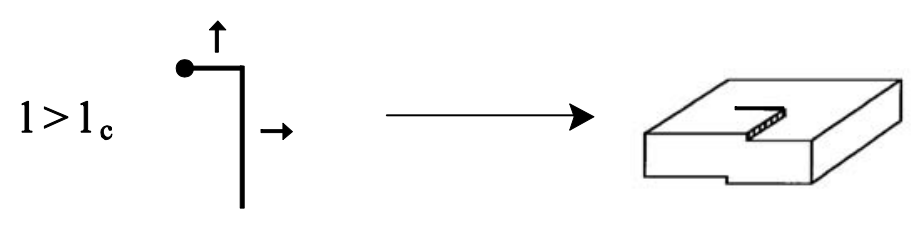

(4)

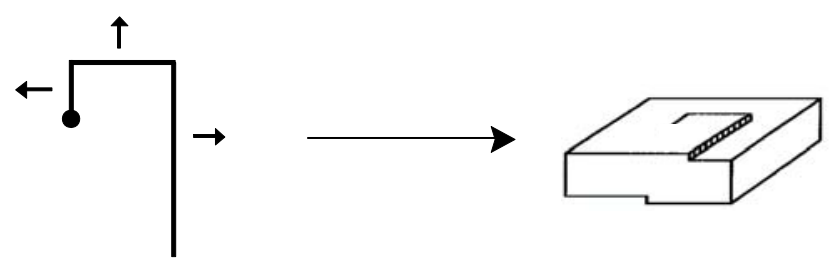

(5)

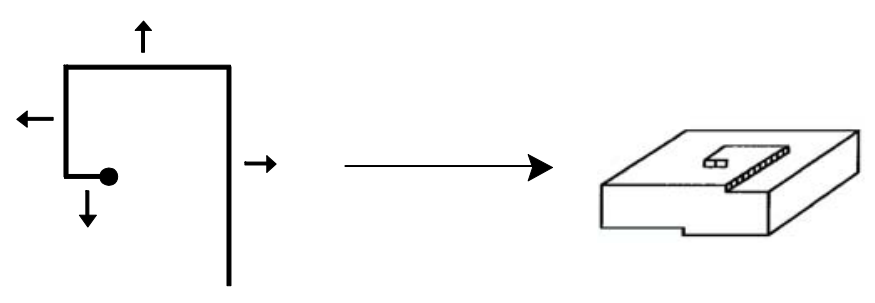

(6)

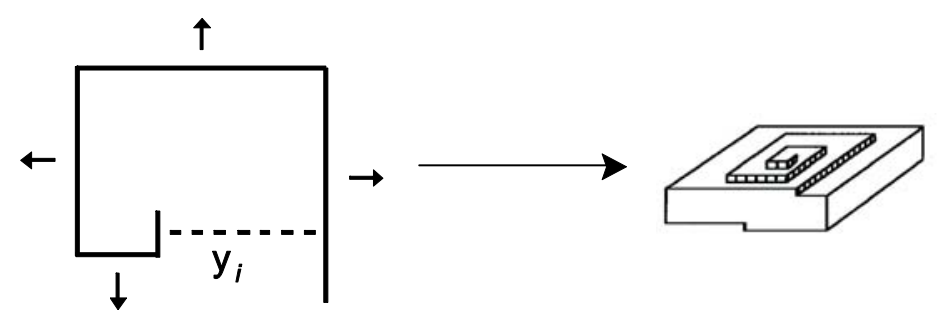

Figure 3.5: The mechanism of spiral growth around a screw dislocation. The initial dislocation edge advances (1) and exposes the adjacent edge, which is stationary while its length is below its critical length, $l_{C}(2)$. The newly exposed edge increases in length due to motion of the preceding step and starts to advance once it has surpassed its critical length (3), exposing the next edge, which in turn advances once it has reached its critical length (4). This process continues (5) until the original edge reappears one layer higher (6). Image adapted and reprinted from Lovette et al. [12] with permission from ACS. 
While the assumption of a Kossel crystal provides a dramatic reduction in complexity, the model of cubic growth units with isotropic interactions perpendicular to each face is insufficient for real crystal systems. Instead, the anisotropic bonding network and facedependent geometry must be considered. The easiest class of real crystals to model is centrosymmetric, where inversion centers present within the lattice force interactions to extend equally in opposite directions. Centrosymmetric crystals have kink sites that are halfcrystal positions, containing the same set of interactions regardless of the specific edge or face.

Non-centrosymmetric crystals contain significant extra complexity from the asymmetric interaction network. Multiple types of kink site can generally exist on each edge [13] and attachment/detachment events, therefore, have different rates at each site. ADDICT is able to make shape predictions for this general class of non-centrosymmetric molecules under the BCF model for spiral growth; the automated implementation of this theory has been the main priority in light of the principal application being API molecules. Extra functionality for the effect of impurities or additional growth mechanisms, such as $2 \mathrm{D}$ nucleation or rough growth, is not currently provided, but could be added as interest and resources dictate. A description of more complicated effects such as solvent entrapment or defect formation appears beyond the current state-of-the-art in mechanistic growth models, so would require further research to enable any description of such effects within ADDICT. A more detailed overview of ADDICT's operation will now be presented. 


\section{ADDICT: a design aid for spiral crystal growth from solution}

In order to empower industrial crystal engineering for the next generation, established mechanistic models must be made as quick to utilize as popular non-mechanistic models. This is our central goal with the development of ADDICT. The software enables rapid predictions without the prerequisite of being an expert in mechanistic modeling, though extra functionality is nonetheless included to deliver additional utility for expert users. Currently, the program is still proof-of-concept software, demonstrating the feasibility of automating mechanistic models for complex molecules. A significant advantage of adopting a mechanistic approach is the ability to refine and assimilate future developments to increase modeling fidelity, an option which does not readily exist for non-mechanistic methods. The software is continually being developed to incorporate new and improved models to extend applicability towards different growth conditions or more complicated systems, based on concurrent research in those areas. This section provides an overview of the ADDICT software, with particular detail in certain areas deemed important to convey an understanding of the program's operation.

\subsection{Outline of ADDICT}

The primary goal of ADDICT is to calculate relative growth rates of the predicted crystal faces (under spiral growth) for a specific solute polymorph and growth environment. Relative growth rates are sufficient to determine the crystal habit using the Frank-Chernov condition (see section 4.6.5).

Since MATLAB is an ideal language to build a user-friendly graphical user interface tool, most codes of ADDICT are written in MATLAB, with some compiled C codes integrated into the program to improve computational efficiency. To deal with ADDICT's complexity 
and manage different types of information, object-oriented program design is applied, where classes are created for various types of information (e.g. lattice symmetry, growth unit/kink/edge/plane properties). A full account of ADDICT's internal operation is beyond the scope of this paper, which will instead provide an overview of its function without addressing finer computational details. The main structure of ADDICT can be divided into 5 modules:

(1) Input parameter preparation. This generates the input panel, which reads the crystallography input alongside all growth conditions and program operation specifications. Partial charges are obtained using external electron density calculation software.

(2) Solid-state interactions. A lattice supercell is generated along with all solid-state interactions using the Generalized Amber Force Field (GAFF) [102] that is applied internally within ADDICT.

(3) Plane and periodic bond chain (PBC) determination. The relevant crystal planes and their corresponding PBCs are determined.

(4) Energetic and mechanistic calculations. The important quantities for the crystal growth model are evaluated (e.g., kink densities, net attachment rates, step velocities etc.) and used to calculate relative growth rates of the crystal faces.

(5) Outputs. ADDICT's results are generated and displayed.

A more detailed explanation of these modules is provided in section 4.6, following specifics relating to modeling PBCs, step edges and growth spirals, the kink rate and solvent effects. 


\subsection{PBC algorithm}

Based on the theory established by Hartman \& Perdok (section 2.2) ADDICT calculates the relative growth rates of $\mathrm{F}$ faces that are important for a particular space group, after applying X-ray extinction conditions. Since the relative growth rates of S and K faces are extremely large, those faces are not considered providing an enclosed crystal can be formed from $\mathrm{F}$ faces alone. If an $\mathrm{S}$ or $\mathrm{K}$ face cannot grow out and lead to a closed solid object a needle-like or plate-like crystal could form, in which case an arbitrarily large relative growth rate is assumed. For each $\mathrm{F}$ face, ADDICT identifies a slice, whose thickness corresponds to the deposited layer for a single elementary growth step.

The Generalized Amber Force Field (GAFF) [102] is used in ADDICT to determine intermolecular interactions, which extended AMBER [103] from proteins to pharmaceutical molecules for use in drug design and should, therefore, be sufficient to accurately describe API molecules. The total interaction is divided into a dispersive and columbic portion using the corresponding GAFF terms, which is required for the solvent effect (see section 4.5).

ADDICT currently works for pure organic molecules, where nearest-neighbor interactions in the crystal dominate the lattice energy due to a lack of significant charges that could result in a larger columbic term in GAFF (the long-ranged portion of the interaction). In ADDICT, an energy cutoff is used in forming PBCs, which can be modified in the input panel. Typically a cutoff of $1 k T$ allows the PBCs to represent at least $90 \%$ of the lattice energy, while exclusively containing nearest neighbor interactions. From this point on the energetics are assumed to be completely represented by the PBCs.

ADDICT calculates the growth of $\mathrm{F}$ faces that are important for a particular space group, based on X-ray extinction conditions. To calculate the intermolecular bonds used to form PBCs, all interatomic interactions between a pair of growth units are summed, with the 
bond vector determined using the center of mass of each growth unit. PBCs are easily determined for centrosymmetric crystal structures, such as naphthalene, since each intermolecular bond corresponds to a PBC: the same bond into a growth unit from one side extends out in the same direction on the other side, with identical energy. This means all PBCs are straight and growth units and kink sites along an edge are identical in terms of energy. For non-centrosymmetric structures, such as paracetamol, lovastatin and RDX (1,3,5Trinitroperhydro-1,3,5-triazine) the determination of PBCs is much more complicated and the constituent bonds of a repeat $\mathrm{PBC}$ unit are in general not all aligned with the overall PBC direction (the PBCs can be somewhat jagged along the overall straight direction). In fitting with their established concept, some properties of PBCs are:

(1) No intermolecular interaction between a pair of growth units can be shared by two PBCs in the same crystal face;

(2) An intermolecular interaction may be shared between two PBCs that are not within the same crystal face;

(3) A stable surface layer must contain two or more PBCs (F face), layers containing one or zero PBC should be considered as unstable layers (S or K faces);

(4) The arrangement of growth units along a PBC direction must have the same stoichiometry as the lattice in that direction;

(5) For non-polar crystal structures (wherein the net dipole moment of the crystallographic unit cell is zero), the component of the electrostatic dipole moment perpendicular to the PBC vector must be zero (this applies to inorganic crystals or organic salts, the modeling of which is planned in a future version of ADDICT) [18];

(6) For polar crystal structures, all the periodic bond chains may have a net perpendicular dipole moment that is parallel to the polar axis direction. However, no mechanistic model is 
currently able to deal with polar crystals so ADDICT cannot handle such cases.

The PBC algorithm for non-centrosymmetric systems in ADDICT uses a combination of hard and "soft" rules in order to optimize the selection of PBCs for each slice. This method could benefit other crystallography researchers, since periodic bond chains are a useful tool in understanding crystal growth and their determination for non-centrosymmetric systems is non-trivial.

Figure 4.1 summarizes the algorithm. Firstly, the slice is defined; the PBC algorithm is applied for each face individually. A list of the lowest Miller index faces is developed and the unique faces in each direction are selected to investigate, from which the slice thickness is defined based on the interplanar spacing (which can be calculated from the Miller indices). X-ray extinction conditions for the crystal polymorph's space group are applied to determine if a reduced interplanar spacing should be selected in any direction, which corresponds to a set of higher Miller indices and can exist for the special directions in the space group lattice where symmetry dictates repeated slices per unit cell as the face grows. 


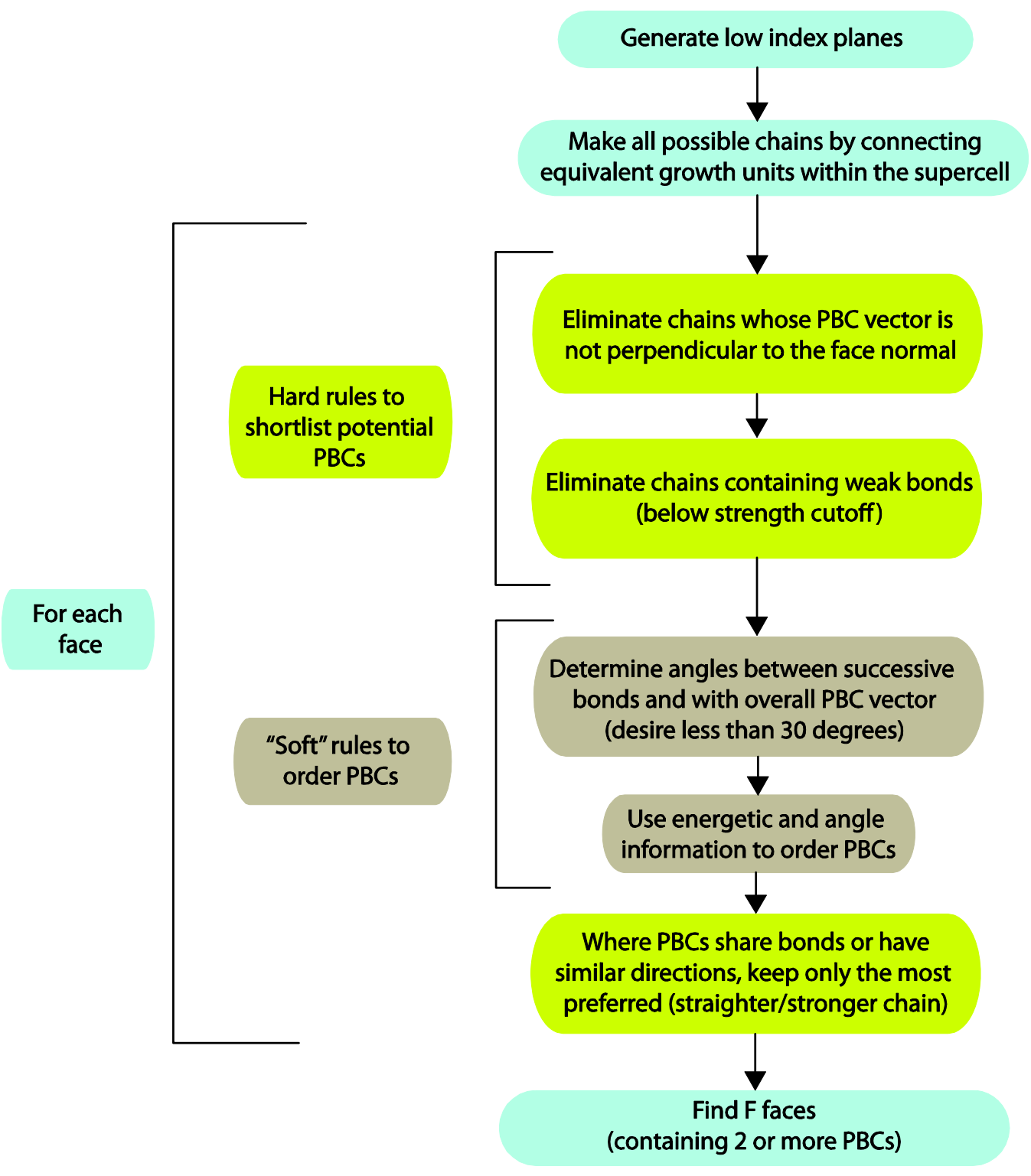

Figure 4.1: Algorithm for finding PBCs and $\mathrm{F}$ faces. 
Secondly, ADDICT generates a list of all possible periodic bond chains. The core concept is that to be periodic, chains must contain a repeat unit which starts and ends on the same growth unit. Chains can, therefore, be generated by considering each growth unit within the crystal and connecting two such growth units by a path of additional growth units. Single connection chains directly connect growth units, 2-connection chains separate growth units by bonding to a different growth unit and so on. Single connection chains are developed by considering each growth unit within the crystal lattice in turn and finding the intermolecular interactions that connect it to itself. In developing 2-connection chains, for each growth unit considered as the start and end points, one must loop through other growth units (and their different positions within the supercell) that can act as an intermediate connection. This strategy is continued for higher connection chains and does not change except in complexity. While forming these chains, one can apply priority rules to determine whether such a chain is remotely physical and warrants further investigation. Firstly, the $\mathrm{PBC}$ vector defining the repeat unit cannot have any component perpendicular to the slice, because upon extending the chain indefinitely it will not remain in the slice. Secondly, chains should not be formed using any intermolecular bonds under the strength cutoff provided in the input panel. This should be of the order $1 k T$ or lower (corresponding to the roughening transition [104-106]) and if no value is provided ADDICT uses $0.2 \mathrm{kcal} / \mathrm{mol}$ by default. These two rules enable the search for candidate PBCs to be reduced to a practical problem using the above approach based on connecting growth units in a periodic fashion.

With the list of candidate chains, ADDICT no longer eliminates but instead ranks PBCs based on "soft" priorities, keeping all the information in order to select the best PBCs for that slice. The desire is to have PBCs correspond to the most stable step edges on a crystal surface, i.e., those sides naturally emerging from a screw dislocation during spiral growth, 
which is why the algorithm effectively finds the most physically stable surface structures. ADDICT treats each spiral itself as a faceted object composed exclusively of those in-slice PBCs. Such straight-sided spirals have been experimentally imaged $[107,108]$ and this picture is essentially exact while the step edges remain below their roughening transition; above this point, step curvature begins to appear. The assumption of straight edges is expected to be valid in the spiral regime and with relative growth rates being calculated (section 4.6.5) errors introduced from this approximation should prove inconsequential.

Alongside chain strength, the main priority in ranking PBCs is to ensure, within the repeat unit, angles between successive bonds are small; $30^{\circ}$ is used as a desirable criterion and the average angle for each chain is calculated in addition to angles between successive bonds (this is an example of a "soft" rule). A final consideration involves a molecular direction descriptor, as shown in Figure 4.2(a) for a paracetamol molecule. This descriptor is found by connecting non-hydrogen atoms on the backbone chain of the molecule, reducing rings to a center of mass. It is expected that favorable PBCs have aligned descriptors for adjacent molecules along the chain, which is another soft rule used for ranking purposes, though one which has lower priority than the need for high interaction strengths and low successive bond angles.

PBCs are then ordered using this strength and straightness information. When bonds are shared or the PBC vector is very similar between multiple PBCs, the chain with higher priority is kept and the other eliminated, leaving unique PBCs that offer the 'best use' of interactions within the slice to form edges with low energy and maximum in-chain interactions.

The slice can be shifted, while maintaining its thickness that is defined by the Miller indices, in order to maximize the slice energy (the contained PBCs). This is an advanced 
technique and often unnecessary, but for complicated non-centrosymmetric cases where the slice is not readily apparent, it can be useful to once again ground the model in an energetically favorable face and edge configuration, which is essential in moving forward for the mechanistic growth calculation to be applicable.

(a)

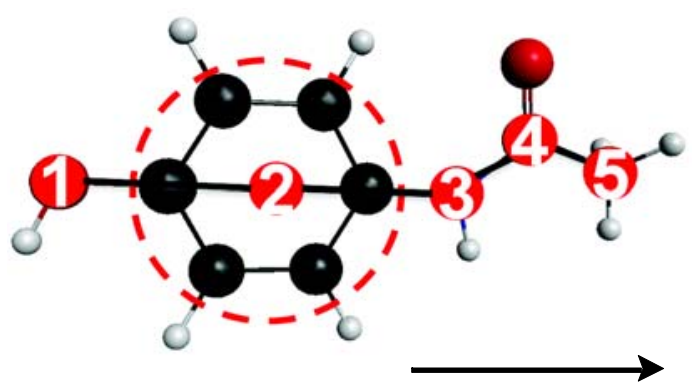

Molecular orientation

(b)
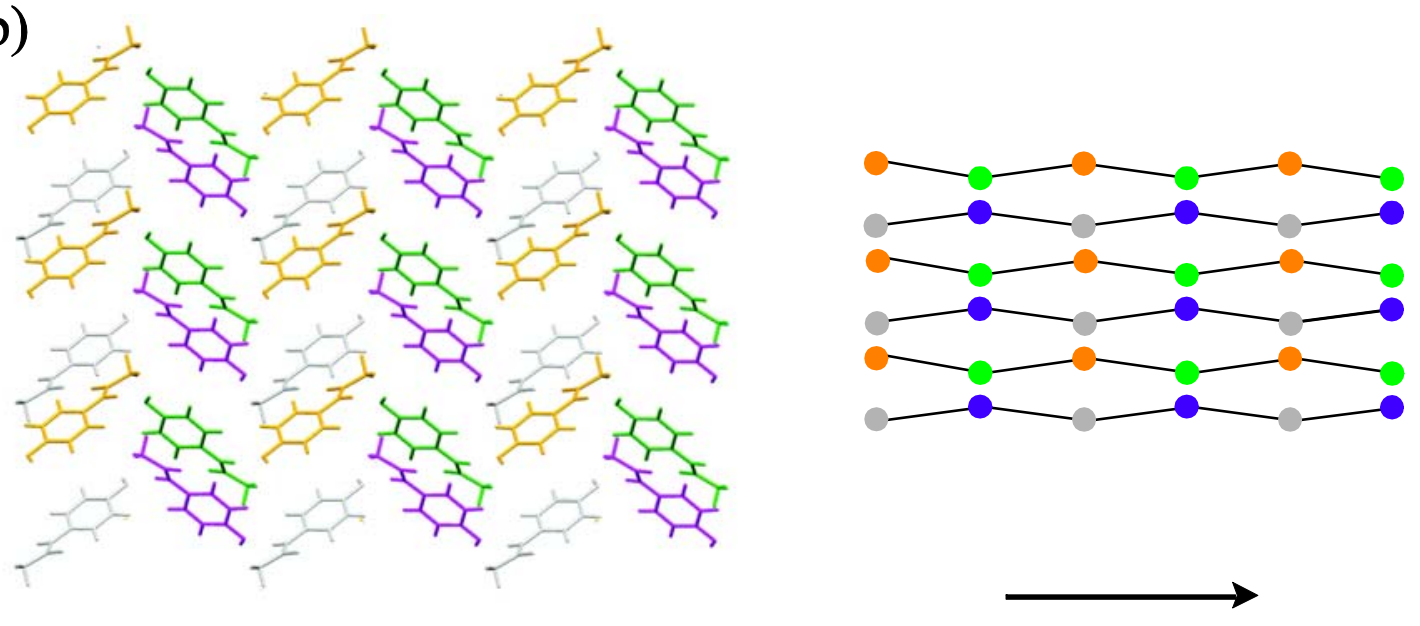

\section{PBC orientation}

Figure 4.2: A molecular direction descriptor for a paracetamol molecule (a) and an example paracetamol plane colored by symmetry operation ((b), left) with the corresponding reduction to point masses and periodic bond chains ((b), right). For a further description of interactions in paracetamol see Kuvadia et al. [13]. 


\subsection{Edges and spirals}

For each spiral turn, as edges emerge from the dislocation, there exists an induction time before an edge begins to advance, because although incorporation of growth units into the lattice is favorable in a supersaturated environment, there is a surface energy penalty at the ends of a spiral edge for addition of a new row. During this induction time the edge is growing due to the advance of the previous spiral edge; hence, there exists a critical length for each exposed edge, which ADDICT calculates based on thermodynamic considerations that find the length at which this surface energy penalty and volume reward are equal $[100,109,110]$. In equation $14, l_{C, i}$ is the critical length of side $i,\left\langle a_{E, i}\right\rangle$ is the average distance

between growth units along the edge and $\left\langle\phi_{k, i}\right\rangle$ is the average kink energy on the edge, which corresponds to the surface energy penalty. These quantities require averaging for noncentrosymmetric systems, where there are usually multiple values on a given edge [13].

$$
l_{c, i}=\frac{2\left\langle a_{E, i}\right\rangle\left\langle\phi_{k, i}\right\rangle}{R T \ln S}
$$

Snyder and Doherty [100] developed an expression for the rotation time of a convex Nsided polygonal spiral (equation 15), which is a summation of the time between each edge being exposed and reaching its critical length (at which point the next edge is exposed); $\alpha_{i, i+1}$ is the angle between edges $i$ and $i+1, l_{C, i+1}$ represents the critical length of the emerging edge $\mathrm{i}+1$ and $v_{i}$ the step velocity of the preceding edge that causes the emerging edge to elongate (note the Voronkov profile [111] is used for $v_{i}$ ). Spiral side disappearance is important [100] and included in ADDICT also, though this will not be described here. 


$$
\tau_{S}=\sum_{i=1}^{N} \frac{l_{C, i+1} \sin \left(\alpha_{i, i+1}\right)}{v_{i}}
$$

The expression for the step velocity of the $i$ th edge is given by equation 16 , where $a_{P, i}, \quad u_{i}$ and $\rho_{i}$ are the distance that the $i$ th edge propagates with the addition of a new row, the net rate of solute attachment to kink sites on the $i$ th edge (kink rate, see section 4.4), and the density of kink sites on the $i$ th edge, respectively [112].

$$
v_{i}=a_{P, i} u_{i} \rho_{i}
$$

The kink density can be described by Boltzmann statistics, where Frenkel [70] developed an expression for centrosymmetric molecules that depends on the kink energy. Hypothetical transformations on the edge can be used to determine the kink energy, such as removing a pair of growth units from one row and depositing them as part of the next row. This process forms 4 kinks, so the kink energy is a quarter of the energetic penalty for this transformation. To calculate a transformation energy cost in vapor growth it is sufficient to consider which PBCs are broken and reformed upon the rearrangement; when a solvent is present the changes in surface energies (see section 4.5) are instead considered to account for the solvent effect.

For non-centrosymmetric molecules, there are multiple possible transformations that each form 4 kinks but of different types, so one cannot extract individual kink energies. Instead, one must evaluate all possible transformations, and then the density of individual kink types is based on a weighted sum of the transformations that can produce that type of kink. The overall kink density is then the sum of these individual kink densities. For the case of the [010] edge on the (001) face of paracetamol (2 successive edges each with 2 growth units), the kink density expression becomes [13]: 


$$
\rho=\left(\frac{\exp \left(-\frac{\left|\varepsilon_{1}\right|}{k T}\right)+\exp \left(-\frac{\left|\varepsilon_{2}\right|}{k T}\right)+\exp \left(-\frac{\left|\varepsilon_{3}\right|}{k T}\right)+\exp \left(-\frac{\left|\varepsilon_{4}\right|}{k T}\right)}{1+\exp \left(-\frac{\left|\varepsilon_{1}\right|}{k T}\right)+\exp \left(-\frac{\left|\varepsilon_{2}\right|}{k T}\right)+\exp \left(-\frac{\left|\varepsilon_{3}\right|}{k T}\right)+\exp \left(-\frac{\left|\varepsilon_{4}\right|}{k T}\right)}\right),
$$

where the $\varepsilon_{i}{ }^{\prime} s$ correspond to energies of the 4 unique transformations, normalized on a per kink basis by dividing the total energy cost of the transformation by 4 . This kink density can be viewed as the Boltzmann probability of each possible transformation existing on the edge.

Non-centrosymmetric crystals also introduce the extra potential complexity of unstable edges. An unstable edge layer exists if the bonds attaching that layer to the step are weaker than those which form upon attachment of a new layer. Therefore, for non-centrosymmetric edge patterns involving unstable layers, there can be a thermodynamic driving force for edge rearrangement and formation of kinks beyond one layer of growth units. This affects transformations and, therefore, the kink density, as well as the order of incorporation into a kink site and hence the kink rate. A method for dealing with these situations has been described by example [13] on a case-by-case basis and ADDICT incorporates the concepts using various possible stable/unstable layer patterns. Nonetheless, this case-by-case approach for kink density and incorporation rate calculations when dealing with non-centrosymmetric edge patterns is not ideal for an automated program such as ADDICT, which occasionally requires modification to deal with new complex patterns not currently included within the program. To address this deficiency, a generalized thermodynamic kink density that universally copes with any edge layer pattern or number of growth units is currently being formulated. 


\subsection{Kink rate model for spiral growth}

In order to obtain step velocities using equation 16 , we must also calculate the kink rate, which is the focus of this section. For non-centrosymmetric molecules this net incorporation rate must consider the various types of kink sites, their individual incorporation rates and the progression between different kink sites as a result of attachment or detachment events. Equation 18 is this general expression for the kink rate $u_{i}$ on a step edge of an organic crystal containing $n$ growth units $[13,19,112]$ ( $n=1$ for centrosymmetric crystals and subscripts $k$ and $i$ correspond to the kink and edge, respectively; note also that the prefactor $n$ was incorrectly omitted in our earlier formulations of equation 18).

$$
u_{i}=n \frac{\left(j^{+}\right)^{n}-\prod_{k=1}^{n} j_{k, i}^{-}}{\sum_{r=1}^{n}\left[\left(j^{+}\right)^{n-r} \sum_{k=1}^{n}\left(j_{k}^{-} j_{k+1}^{-} \cdots j_{k+r-2}^{-}\right)_{i}\right]}
$$

The attachment rate, $j^{+}$, is identical for each kink site, assuming isotropic desolvation effects (see equation 19). The detachment rate, $j_{k, i}^{-}$, is instead specific to kink $k$ on edge $i$ due to dependence on the work of kink detachment (see equation 20). For a centrosymmetric crystal the kink rate on an edge (dropping the ' $i$ ' subscript) reduces to $j^{+}-j^{-}$, where kink sites are identical due to the PBC symmetry. In non-centrosymmetric systems, one must substitute equations 19 and 20 for each kink site into equation 18 . Different growth units exist on an edge due to the various lattice orientations upon incorporation and furthermore whether the kink for a specific growth unit is in an 'east' or 'west' orientation on the step edge affects its detachment work since different PBC bonds will usually exist on either side in a non-centrosymmetric system. Equation 18 is for the kink rate in a single direction, i.e., either 'east' or 'west' kinks alone. 
We use transition state theory (TST) [113] to develop expressions for the attachment and detachment rates to use within equation 18. We expect organic molecular crystals (with fast surface diffusion) to grow via a 3-event mechanism: terrace adsorption, step adsorption and finally kink incorporation; inorganic crystals are conversely expected to incorporate directly from solution in light of slower surface diffusion. A microkinetic model can be formulated to describe each process within a 3-event mechanism; the resulting kink rate for centrosymmetric crystals is essentially the same as if a single-event mechanism of incorporation directly from solution is adopted [76]. Thus, although incorporation into a kink is expected to be preceded by adsorption onto a step, rate expressions can be simplified by assuming a single-event mechanism. Figure 4.3 demonstrates the resulting free energy picture. At state $\mathrm{S}$ both the kink site and growth unit in solution are solvated. The transition state corresponds to partial desolvation of both entities once the growth unit has approached the kink site. State $\mathrm{X}$ corresponds to the growth unit incorporated into the kink with the surface re-solvated. The barrier $\Delta G^{\dagger}$ indicates that this is an activated process, while the free energy difference $G_{S}-G_{X}$ captures the driving force for crystallization (at equilibrium this difference is zero and there is no crystal growth or dissolution). A reaction coordinate could involve the distance from the adsorbed growth unit to its corresponding position upon kink site incorporation (e.g., Stack et al. [20], who consider this distance in 3 dimensions), but additional descriptors are expected to be necessary in general (to account for the solvent etc.).

The resulting attachment and detachment rates from TST are:

$$
\begin{gathered}
j^{+}=v_{0} \exp \left(-\frac{\Delta G^{\dagger}}{k_{B} T}\right) x \\
j_{k, i}^{-}=v_{0} \exp \left(-\frac{\Delta G^{\dagger}+\Delta W_{k, i}}{k_{B} T}\right)
\end{gathered}
$$




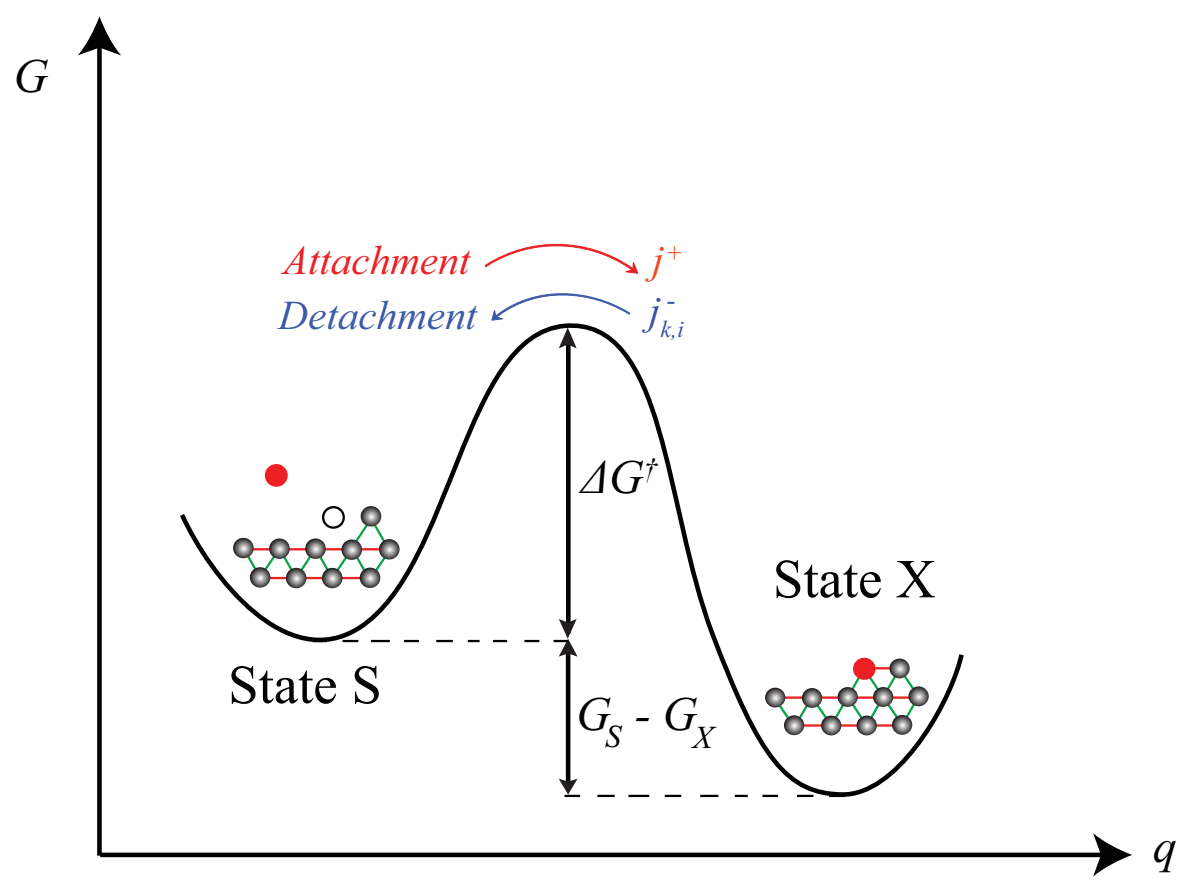

Figure 4.3: Schematic free energy diagram for the attachment and detachment of solute growth units from kink sites. States $\mathrm{S}$ and $\mathrm{X}$ represent growth units in solution and incorporated to the crystal, respectively; $j^{+}$and $j_{k, i}^{-}$are the corresponding attachment and detachment rates. A simplified reaction coordinate $q$ could relate to the distance of the growth unit from the kink site. The filled red circles represent the growth unit which is attaching to/detaching from the kink site and the open black circle represents the unoccupied kink site.

Thus, the attachment rate at a kink site is treated as first order in solute concentration in solution ( $x$; this introduces a dependence on supersaturation, ensuring that a higher crystallization driving force increases the attachment rate), while the detachment rate is zeroth order. Compared to rate expressions within the previous kink rate model [112], the detachment rate no longer includes a prefactor of the solvent (or solvation-assisting species) mole fraction. This aligns with crystallization thermodynamics, which will be discussed in a forthcoming paper. To enable rapid computation, the standard free energy change of crystallization within $j_{k, i}^{-}$is calculated as an energetic quantity, $\Delta W_{k, i}$ (the detachment work 
from kink $k$ on edge $i$ ), which assumes entropic changes are negligible (this is most justifiable for small molecules [67]). In the vapor, $\Delta W_{k, i}$ corresponds to the PBCs that must be broken to detach a kink growth unit from the lattice, while in solution it corresponds to the change in surface energies, which includes breaking PBCs and solvation of surfaces.

The barrier $\Delta G^{\dagger}$ corresponds to partial desolvation of both the kink site and attaching growth unit before incorporation, under solution growth. From the vapor, this effect is absent so the barrier might be more entropic in nature, as the solute growth unit must orient properly before incorporation. The only reliable way to calculate this quantity is to use molecular simulations. Stack et al. have demonstrated this for the incorporation of a barium ion at a kink on a barite $\left(\mathrm{BaSO}_{4}\right)$ surface [20], where they calculated the free-energy surface for the attachment process. This method identifies various intermediate states with varying degrees of desolvation and enables one to identify the correct rate-determining process and model the mechanism appropriately. The barrier $\Delta G^{\dagger}$ is assumed to be isotropic and common to all kink sites on each edge and face, as a first approximation (the frequency prefactor $v_{0}$ is additionally assumed isotropic, which has been done with success elsewhere [114]). Therefore, both quantities cancel upon the formation of relative growth rates, enabling shape (not size) predictions without the need to estimate $\Delta G^{\dagger}$ or $v_{0}$, which would not be feasible on a computational timescale appropriate for ADDICT.

The effect of external species such as structurally similar additives has been studied within the context of the mechanistic model [115]; however at present the ability to account for such effects has yet to be built into ADDICT.

Using equations 14-20, the spiral rotation time can be predicted for each face. This enables the prediction of relative growth rates and the crystal shape, which is described in section 4.6. 


\subsection{Solvent effects}

The choice of solvent is a critical design decision and can significantly affect the crystal morphology. The solvent's presence during crystallization modifies surface energies due to solvation of the crystal surface, impacting kink densities and rates. The most accurate way of accounting for this solvent-induced energetic modification would be molecular simulations [20,114], but ADDICT requires a far shorter computational timescale in order to remain a practical tool (hence why the estimation of $\Delta G^{\dagger}$ and $v_{0}$ would be similarly impractical). Instead, to calculate the solvent effect, the bulk interface approximation is used (equation 21) $[5,7,100]$ where to create a surface the internal crystalline and solvent cohesive interactions must be broken and then solvent-crystal bonds can reform (adhesion work). Subscripts 's' and ' $X$ ' refer to solvent and crystal and superscripts ' $d$ ' and ' $A B$ ' refer to dispersive and acidbase, respectively.

$$
\gamma_{X S}=\left(\gamma_{X}^{d}+\gamma_{X}^{A B}\right)+\left(\gamma_{S}^{d}+\gamma_{S}^{A B}\right)-\left(W_{a d, X S}^{d}+W_{a d, X S}^{A B}\right)
$$

The work of adhesion is calculated in one of two ways, according to whether the crystalsolvent interaction has any acid-base character or not (hydrogen bonds are the most ubiquitous example). ADDICT will attempt to find locations of acid-base interactions by finding those PBCs with a large columbic character in GAFF. This approach was prompted by a recent investigation into the most appropriate way to model solution effects within the mechanistic crystal growth model; a brief summary of the implementation will be presented here with more details appearing in an upcoming paper [76].

Assuming the presence of an acid-base interaction, the dispersive and acid-base components of the adhesion work are calculated using equations $22 \& 23$ following the model 
by van Oss, Chaudhury \& Good [116,117] which uses a geometric mean for the dispersive contributions and separates the acid-base portion of the adhesive effect into two parts that match donating to accepting characteristics between the crystal and solvent.

$$
\begin{gathered}
W_{a d, X S}^{d}=2 \sqrt{\gamma_{X}^{d} \gamma_{S}^{d}} \\
W_{a d, X S}^{A B}=2 \sqrt{\gamma_{X}^{+} \gamma_{S}^{-}}+2 \sqrt{\gamma_{X}^{-} \gamma_{S}^{+}}
\end{gathered}
$$

Equation (24) describes the partitioning of interactions for phase $i$ within this model; superscripts $+\&-$ refer to accepting (Lewis acid) and donating (Lewis base) characteristics, respectively.

$$
\gamma_{i}=\gamma_{i}^{d}+\gamma_{i}^{A B} \quad \gamma_{i}^{A B}=2 \sqrt{\gamma_{i}^{+} \gamma_{i}^{-}}
$$

On the crystal side, surface energies are calculated by summing the PBCs extending out of the relevant surface (kink and edge energies are those required by the model) and dividing by the appropriate area. The dispersive portion $\left(\gamma_{X}^{d}\right)$ is calculated by multiplying the total PBC interaction strengths by $d /(d+c)$, where $d$ and $c$ correspond to the $r^{-6}$ and $r^{-1}$ terms from GAFF, respectively. The acid-base portion $\left(\gamma_{X}^{A B}\right)$ is calculated by multiplying instead by $c /(d+c)$. This fractional treatment is used because partitioned force field terms are less reliable than the total interaction energy [118].

On the solvent side, Hansen's 3-component solubility parameters [119] are used in combination with Beerbower correlations [120]. Three correlations exist for different classes 
of materials, but upon application of Hansen parameters for the chosen solvent, an empirical prefactor is used to scale the resulting predicted surface energy to match an experimental value (correcting for temperature effects). Following this, $\gamma_{S}^{d}$ is obtained by extracting the dispersive solubility parameter $\left(\delta_{d}\right)$ term. Then $\gamma_{S}^{A B}$ is obtained by subtraction from the total surface energy. This avoids the questionable subdivision into polarization and hydrogenbonding solubility parameters [121].

Therefore, both crystal and solvent interactions can be partitioned into dispersive and acid-base components. Further subdivision of the acid-base component into donating and accepting parts requires an assumption of the $\gamma^{+} / \gamma^{-}$ratio, unless tabulated values [122] exist for the molecules involved or for those with similar functional groups. Nonetheless, PBCs with large acid-base character (that can interface with the solvent) often grow out of spiral edges due to this larger work of adhesion and faster step velocity; therefore, shape predictions can be insensitive to the assumed value of this ratio [76]. The user can change the value of this $\gamma^{+} / \gamma^{-}$ratio (for both solute and solvent) to be used within ADDICT, permitting a sensitivity analysis to observe the effect, if any, on the overall crystal shape. This can allow the user to understand when more complicated solution effects may be present and in that case use predictions in a more qualitative manner.

When there are negligible acid-base interactions, the work of adhesion is modified to equation 25, since dispersive forces now dominate adhesion. The total PBC strength is used for the crystal energy, since its acid-base character is negligible and this is matched against the dispersive energy from the solvent.

$$
W_{a d, X S}=2 \sqrt{\gamma_{X} \gamma_{S}^{d}}
$$


In summary, this method involves a specific application of interfacial effects as appropriate. Best results are obtained for purely dispersive systems, without moleculespecific acid-base effects. Amphoteric crystal/solvent cases can be dealt with relatively well, where a $\gamma^{+} / \gamma^{-}$ratio close to unity is a reasonable assumption. This approach would require modification for monopolar systems of opposite character however, where internal crystal and solvent interactions have no acid-base self-association but upon contacting each other hydrogen bonds or other acid-base interactions can form.

\subsection{A detailed blueprint of ADDICT}

A blueprint of ADDICT is shown in Figure 4.4. This is in essence a more detailed picture of the module organization already presented in section 4.1 . The colored sections segregate according to functionality, each of which will now be discussed in more detail. 


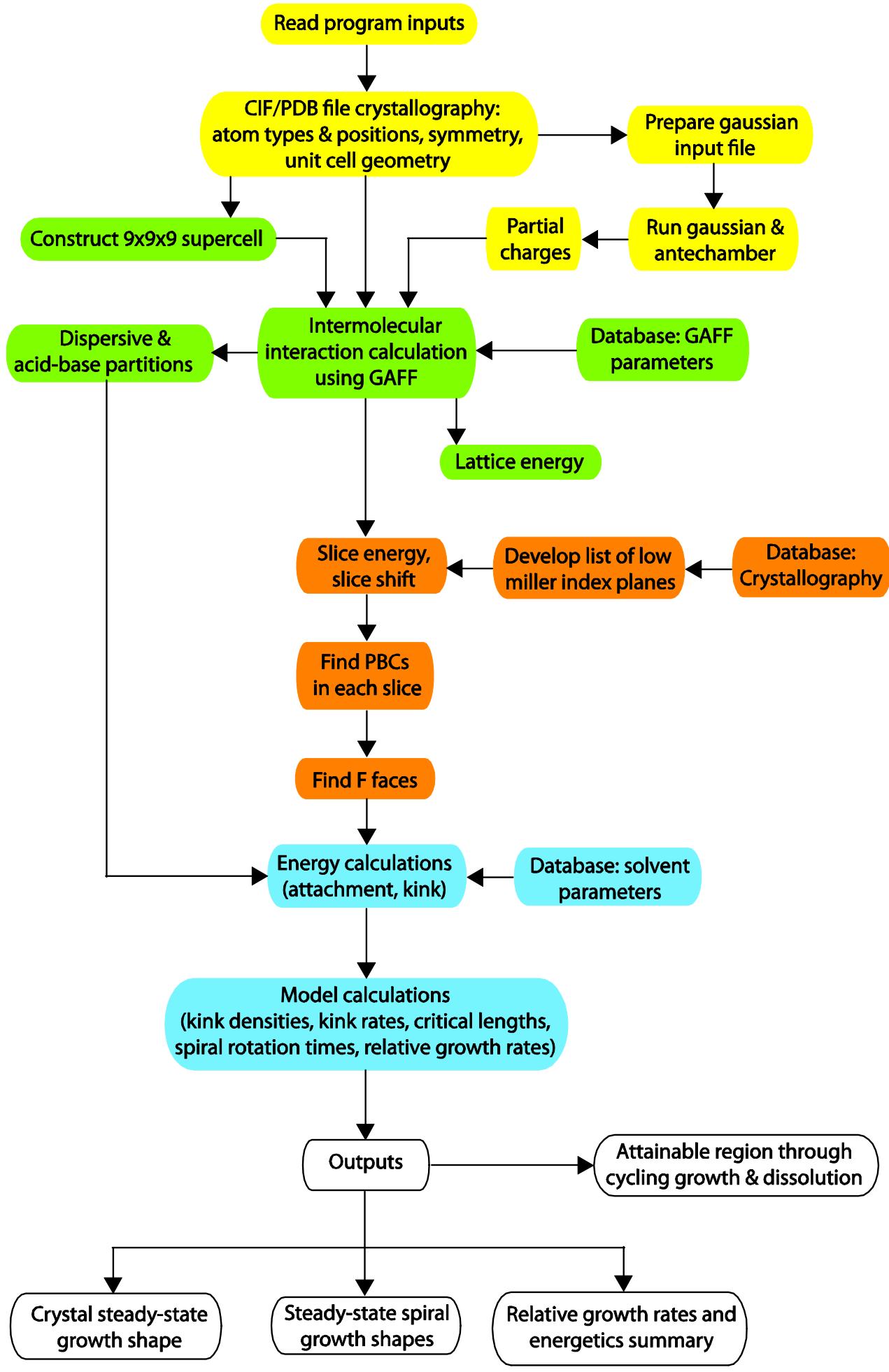

Figure 4.4: Schematic diagram for the structure of ADDICT. 


\subsubsection{Input parameter preparation (yellow)}

The input panel in ADDICT reads specifications of the crystal system, growth conditions (temperature, solvent, supersaturation) and desired program functionality. One can browse for the crystallography input file and select a directory to save the results. Crystallography data for the desired polymorph (e.g., as obtained from X-ray diffraction) can be provided in a CIF (Crystallographic Information File) or PDB (Protein Data Bank) file format and 194 space groups are so far compatible with ADDICT. The user may select either a monomer or dimer growth unit; when a dimer is selected ADDICT treats interactions between the constituent dimer growth units as intramolecular and ignores them, while all the relevant intermolecular interactions between separate dimer pairs are used to calculate inter-dimer interactions.

Cell lengths \& angles, atoms types \& positions and symmetry operations are read, stored and used to generate a Gaussian 09 input file (.com), which must be used to perform an electron density calculation with the commercial software [123]. The default functional and basis set in the .com file are B3LYP/6-31G*, which could be adjusted by an expert user. Antechamber from AmberTools (open source software) [124] must then be run, using the Gaussian output to calculate restrained electrostatic potential (RESP) charges, for a more accurate description of the electron density than the Mulliken point charges calculated using Gaussian. The output from Antechamber is a .mol2 file which should be added to the same directory as the CIF/PDB file before continuing to run ADDICT.

\subsubsection{Solid-state interactions (green)}

From the crystallography, symmetry operations are applied to the asymmetric unit to generate the unit cell, which is translated in the crystallographic directions to form a $9 \times 9 \times 9$ supercell. Intermolecular interactions within the supercell are then calculated using the GAFF parameter database within ADDICT and the obtained RESP point charges; Kirkwood-Slater 
mixing rules are used to determine atom-atom parameters using polarizabilities [125]. A cutoff distance beyond which interactions are neglected is determined by considering lattice energy convergence. If an intermolecular interaction is included, even interatomic interactions with distances larger than the cutoff must be included to ensure there are no false columbic artifacts from the atomic partial charges. The total interaction strengths are stored, alongside dispersive $\left(r^{-6}\right)$ and columbic $\left(r^{-1}\right)$ portions in order to appropriately modify energetics in accounting for the solvent effect later. Users can compare the calculated lattice energy to experimentally determined sublimation enthalpies, where available. If there exists significant difference (i.e., greater than $\sim 4$ RT), users could select an alternative CIF file reported for the same polymorph or perform a structure optimization to generate a new crystallography input; if lattice and sublimation energies can be more closely aligned this provides an improved description of solid-state interactions.

\subsubsection{Plane and PBC determination (orange)}

As described in section 4.2, a list of low Miller index planes is developed according to the crystal space group, alongside the most stable slice for each; following this, PBCs are determined for each slice.

The default slice shift setting is 'Auto Slice', where the selected number of displacements (4 by default) is generated to shift the slice incrementally from its initial position up to a single interplanar spacing. If 'No Slice' was selected then no shifting occurs and the 'UserDefined' option allows specification of both the number of slices and the displacement between each slice (removing the constraint of reaching a displacement of one interplanar spacing). The highest slice energy configuration is selected, but as mentioned previously, this is an advanced technique that is often not required. 


\subsubsection{Energetic and mechanistic calculations (blue)}

This section calculates all the parameters required for the mechanistic growth model. Relevant energies (e.g. PBCs \& kink energies) are calculated, applying the modifications (section 4.5) for the chosen solvent (parameters for 31 solvents are presently contained within ADDICT). The spiral rotation time on each face is determined, using the appropriate crystallographic geometry and relevant quantities and techniques detailed in sections $4.3 \&$ 4.4.

With the growth rate of a face expressed as $G=h / \tau_{s}$, the relative growth rates of $\mathrm{F}$ faces (with reference to the slowest-growing facet) are then calculated, to remove the need to estimate $\Delta G^{\dagger} \& v_{0}$ as discussed in Section 4.4. Absolute growth rates cannot be calculated without estimates for these uncertain parameters, hence our predictions are for the crystal shape, not size.

\subsubsection{Outputs (white)}

From the relative growth rates, one can predict the steady-state crystal morphology by recasting the Frank-Chernov condition [33,34] (Figure 4.5) to:

$$
\frac{R_{1}}{X_{1}}=\frac{R_{2}}{X_{2}}=\frac{R_{3}}{X_{3}}=\frac{R_{n-1}}{X_{n-1}}=1
$$

where $R_{i}=G_{i} / G_{n}$ is the growth rate of face $i$ relative to a reference face $\mathrm{n}$ and $X_{i}=H_{i} / H_{n}$ is the perpendicular distance of face $i$ from the crystal center relative to the corresponding distance for face $n$. The $X$ values are used in ADDICT to construct the steady-state growth shape of the crystal.

Identifying the $\mathrm{PBC}$ directions and step velocities allows one to predict the shape of the 
growth spirals formed on each of the F faces present on a crystal surface. For each of the F faces, a detailed spiral step velocity calculation using kink densities and kink rates is carried out. On the basis of the crystal geometry and the spiral edge velocity calculation, the relative growth rates of all $\mathrm{F}$ faces can be calculated, and the steady-state crystal morphology can be constructed by using Equation 26.

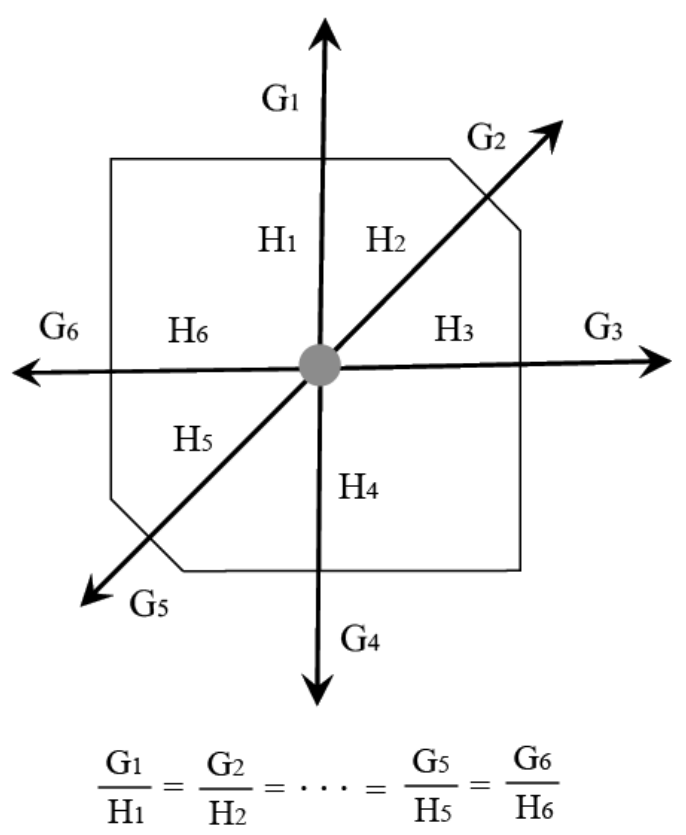

Figure 4.5: The Frank-Chernov condition to predict the steady-state growth morphology, considering a cross-sectional view of the crystal showing $6 \mathrm{~F}$ faces, where $G_{i}$ is the growth rate of face $i$ and $H_{i}$ is the perpendicular distance of face $i$ from the center of the crystal.

Upon running ADDICT, output windows appear with the calculation results. The steadystate crystal growth shape is rendered, alongside spiral shapes on each face. Relative growth rates and relevant energetics are also available and saved in the output folder. The attainable region sub-module allows the user to discover the range of possible shapes through cycles of growth and dissolution [126]. 


\section{Predictions using ADDICT}

ADDICT has been used to study the crystal growth of many organic molecules, both centrosymmetric and non-centrosymmetric, grown from a variety of solvents. In the next several subsections, some examples of tested crystal systems are presented, including: naphthalene, anthracene, paracetamol, lovastatin, D-mannitol, and $\alpha$-glycine.

\subsection{Case study: Naphthalene}

The simplest polycyclic aromatic hydrocarbon, Naphthalene (formula $\mathrm{C}_{10} \mathrm{H}_{8}$ and a white solid) is an example of a centrosymmetric crystal system. Naphthalene crystallizes in the monoclinic space group $\mathrm{P} 2{ }_{1} / \mathrm{a}$ with two molecules per unit cell and the following lattice parameters: $a=8.213 \AA ; \mathrm{b}=5.973 \AA ; \mathrm{c}=8.675 \AA ; \alpha=\gamma=90^{\circ}$; and $\beta=123.388^{\circ}$ [127]. Figure 5.1 portrays the overall methodology for predicting the crystal morphology of naphthalene; (a) shows the crystallography, (b) shows the periodic bond chain networks for the F faces, (c) displays their predicted growth spirals, and (d) indicates the steady-state crystal shape prediction.

The families of $\mathrm{F}$ faces that appear on the crystal habit of naphthalene are $\{001\}$, $\{1 \overline{1}\},\{110\}$ and $\{20 \overline{1}\}$. Figure 5.2 is a summary of ADDICT's predictions for vapor and solution growth from ethanol and cyclohexane. It can be seen that both the spiral shapes on each face and the overall crystal shapes depend on the choice of solvent. ADDICT's predictions for the steady-state crystal growth shape are in agreement with experimental results for both vapor and solution growth [128]. 


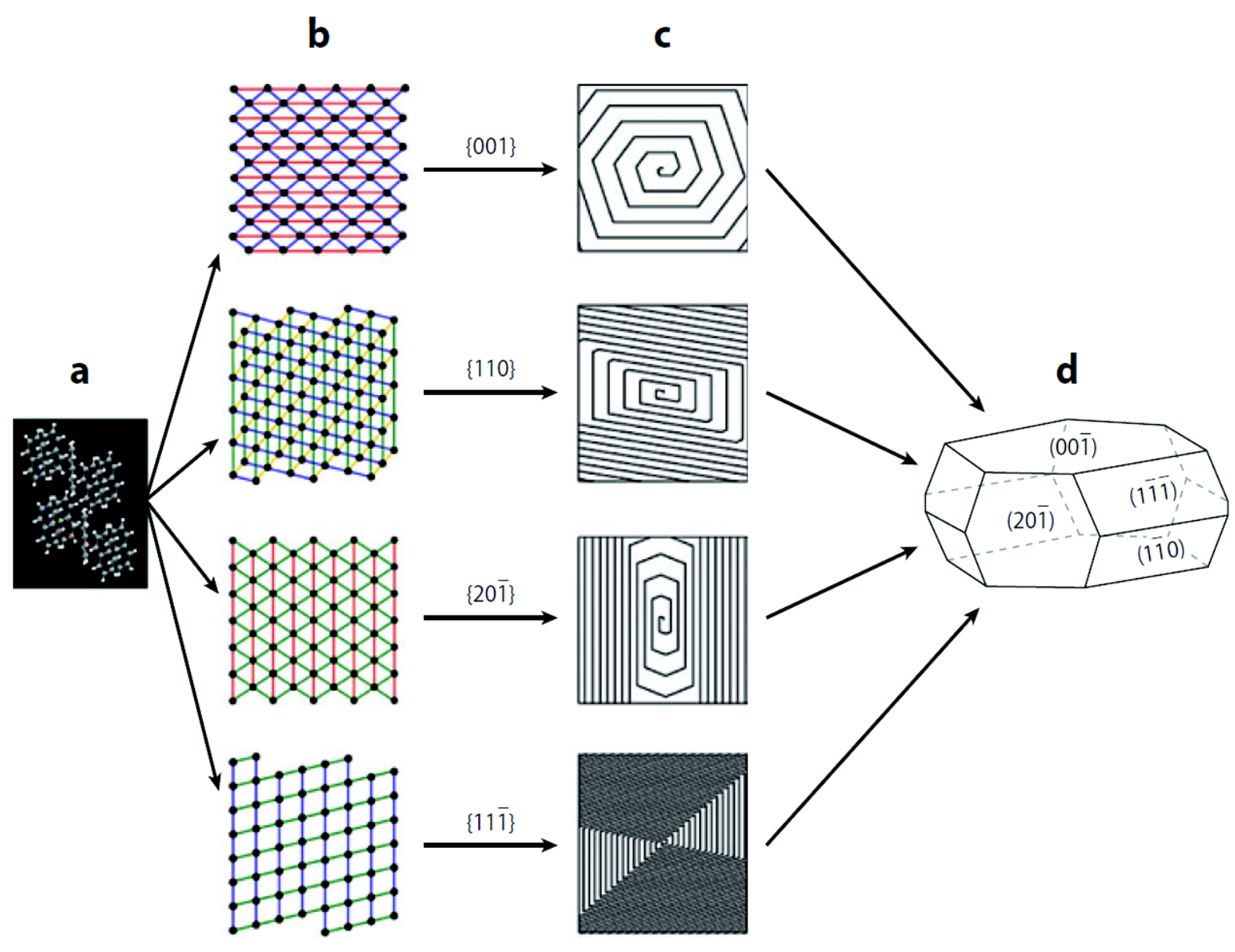

Figure 5.1: The overall methodology for predicting the crystal shape of naphthalene (shown here for growth from ethanol): the crystallography is an input (a); the periodic bond chain network on each face is then determined (b); step velocities and spiral shapes/rotation times are calculated (c); and the steady-state growth morphology results from the relative growth rates (d). Adapted from image courtesy of M.A. Lovette (private communication). 


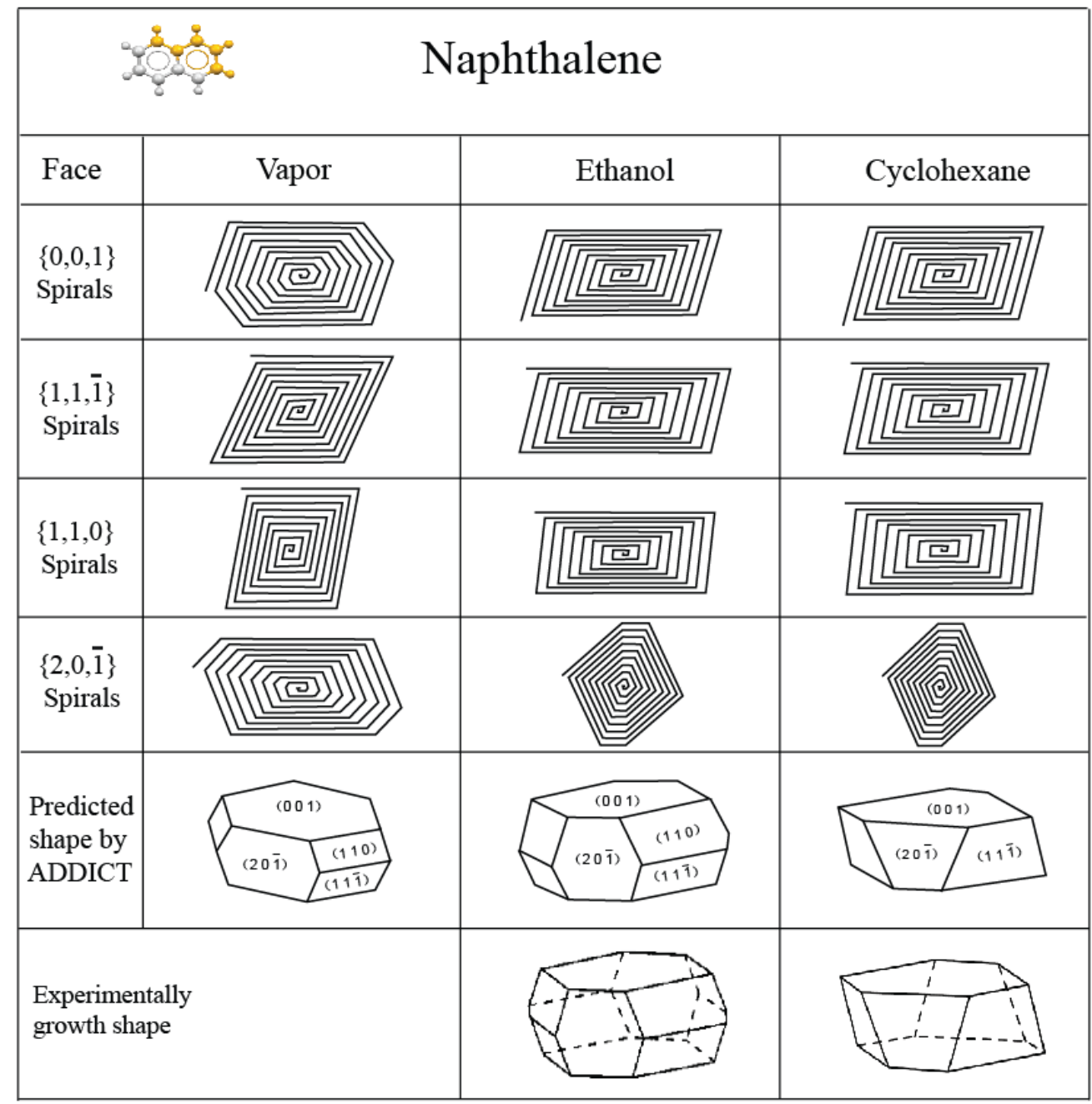

Figure 5.2: A summary of ADDICT's predictions for the growth of naphthalene (Mercury [39] image top left) from the vapor and from solutions of ethanol and cyclohexane. The spiral shapes for the dominant faces are displayed for comparison, alongside the steady-state crystal growth habits, which compare well with experimental results obtained by Grimbergen et al. [128]. 


\section{Anthracene}
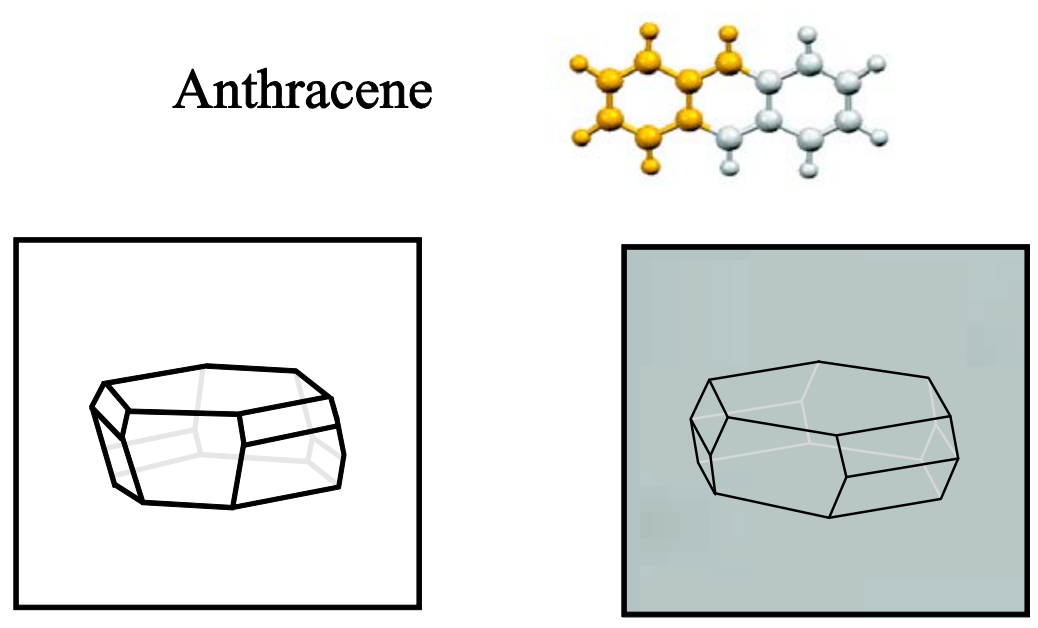

(a) Predicted shape of anthracene in Vapor
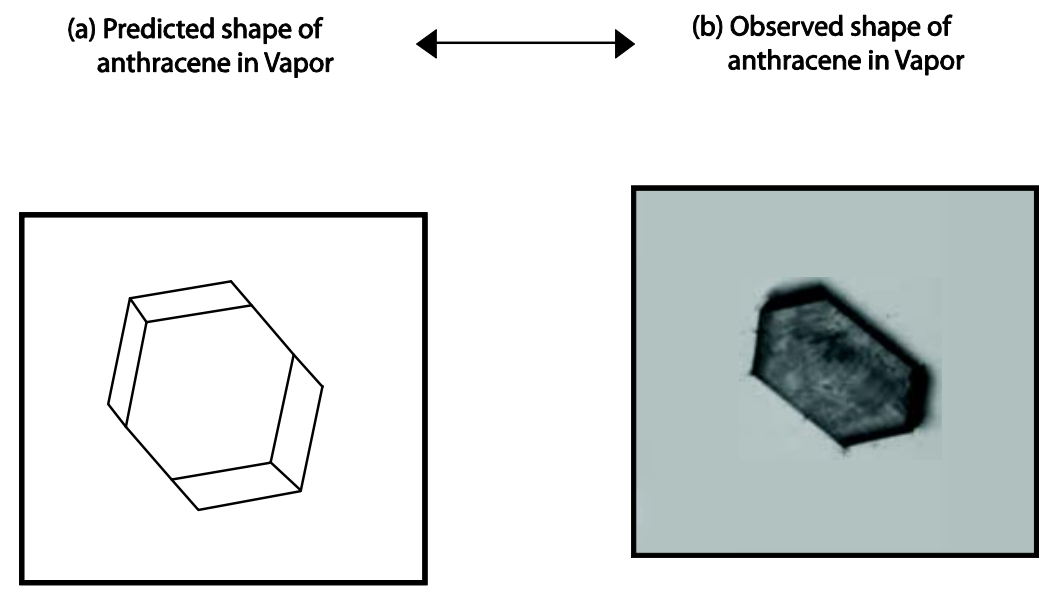

(c) Predicted shape of anthracene in 2-propanol

(d) Observed shape of anthracene in 2-propanol

Figure 5.3: The molecular structure of anthracene (top; image produced with Mercury [39]); a comparison between (a) the predicted vapor-growth shape of anthracene using ADDICT and (b) the experimentally reported vapor shape (schematic traced from Grimbergen et al. [128]); a comparison between (c) the predicted shape of anthracene grown from 2-propanol and (d) the corresponding experimental shape [100]. 


\subsection{Case study: Anthracene}

Anthracene (Figure 5.3, formula $\mathrm{C}_{14} \mathrm{H}_{10}$ ) is another polycyclic aromatic hydrocarbon. It crystallizes in the monoclinic space group $\mathrm{P} 2{ }_{1} / \mathrm{a}$ with the following lattice parameters: $a=8.562 \AA ; \mathrm{b}=6.038 \AA ; \mathrm{c}=11.184 \AA ; \alpha=\gamma=90^{\circ} ;$ and $\beta=124.7^{\circ}$ [129]. There are two molecules per unit cell located at the unit cell [uvw] positions of $(0,0,0)$ and $(1 / 2,1 / 2,0)$. The sublimation crystallization of anthracene has been studied previously using both the attachment energy method [128,130] and Monte Carlo simulations [131]. The predicted sublimation growth shape of anthracene using ADDICT is shown in Figure 5.3 and compares well with the experimentally observed shape [128]. Solution growth from 2-propanol has additionally been studied and again ADDICT's predicted morphology agrees well with experimental determinations (see Figure 5.3). The predicted families of $\mathrm{F}$ faces are: $\{001\}$, $\{1 \overline{1}\},\{110\},\{20 \overline{1}\}$ and $\{200\}$.

\subsection{Case study: Paracetamol}

Paracetamol (acetaminophen) is a complex organic molecule that is non-centrosymmetric, leading to multiple types of kink sites on each face and step edge (for example, the [010] edge of the $\{001\}$ face contains four different kink sites) [13]. Various experimental and theoretical approaches have been conducted to predict the crystal growth of paracetamol and the effect of different external conditions on both the growth process and the steady state morphology $[13,63,112,132,133]$.

Paracetamol crystallizes in the space group $\mathrm{P} 2{ }_{1} / \mathrm{a}$ with lattice parameters: $a=12.92 \AA ; \mathrm{b}=9.4 \AA ; \mathrm{c}=7.1 \AA ; \alpha=\gamma=90^{\circ}$; and $\beta=125.9^{\circ}[134]$. The relevant families of $\mathrm{F}$ faces 
for paracetamol are $\{110\},\{011\},\{200\},\{20 \overline{1}\},\{001\}$ and $\{020\}$. Figure 5.4 shows the predicted spiral shapes under water growth for these faces alongside two AFM images for a growth spiral on the $\{001\}$ face of a paracetamol crystal grown from water; this aligns with the predicted shape and the AFM depth profile confirms the assumption of an elementary step of the calculated interplanar spacing, $d_{001}$. Figure 5.5 compares predicted paracetamol morphologies for vapor and water growth against experimentally observed shapes [135], indicating excellent agreement. Further information about predictions for paracetamol using ADDICT can be found in a previous paper [112], where the steady-state morphologies of paracetamol grown from 30 different common solvents are reported. 
(a)

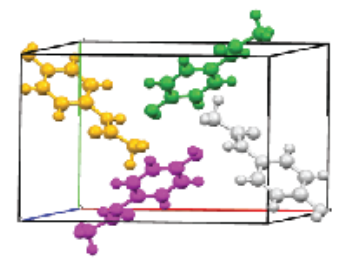

The unit cell of paracetamol

(b)

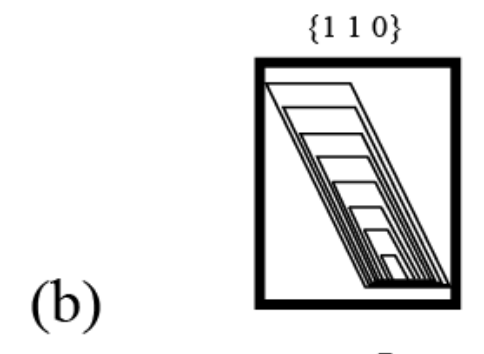

$\left\{\begin{array}{lll}0 & 1 & 1\end{array}\right\}$

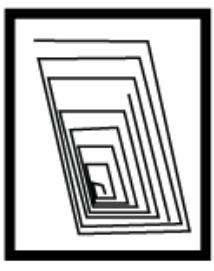

$\left\{\begin{array}{lll}2 & 0 & 0\end{array}\right\}$
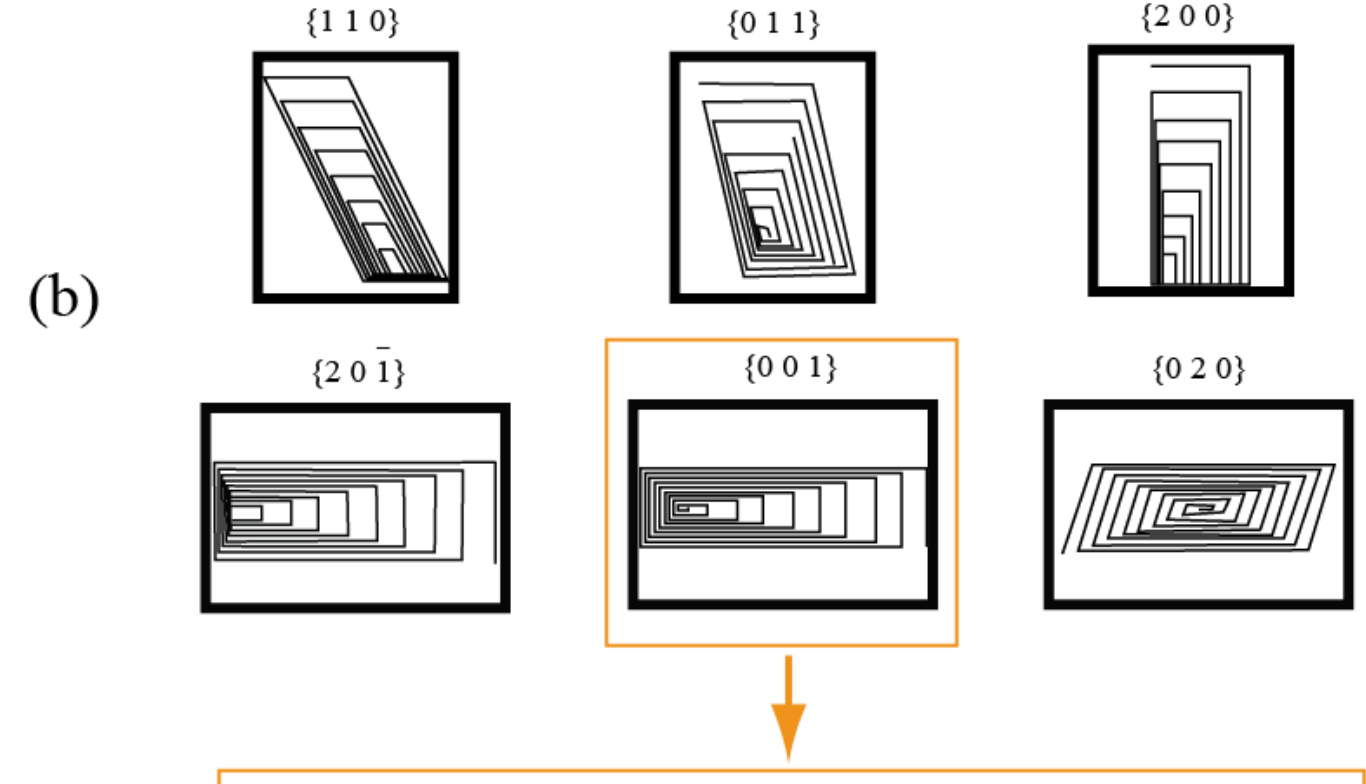

(c)

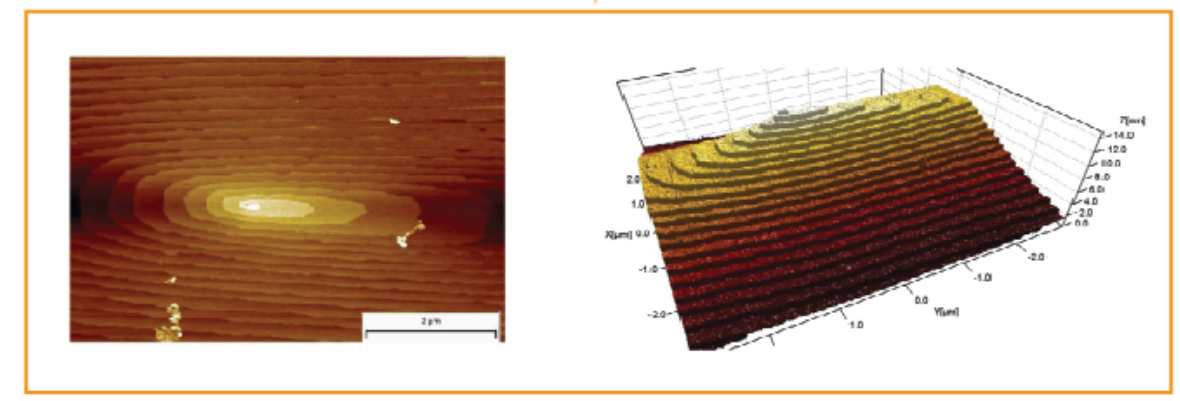

Figure 5.4: (a) The unit cell of paracetamol (image produced with Mercury [39], Z=4, J=1); (b) predicted spiral shapes using ADDICT on all the relevant families of $\mathrm{F}$ faces for paracetamol grown from water; (c) experimental AFM images of a (001) aqueous growth spiral. Figure 5.4 (c) is reprinted with permission from AlChE Journal [136]. 


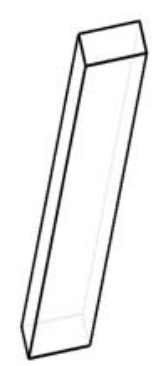

(a) Predicted shape of paracetamol in Vapor

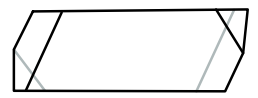

(c) Predicted shape of paracetamol in Water

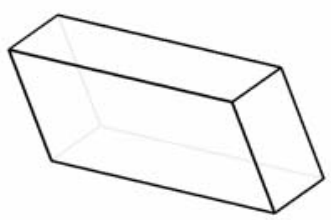

(e) Predicted shape of paracetamol in Methanol

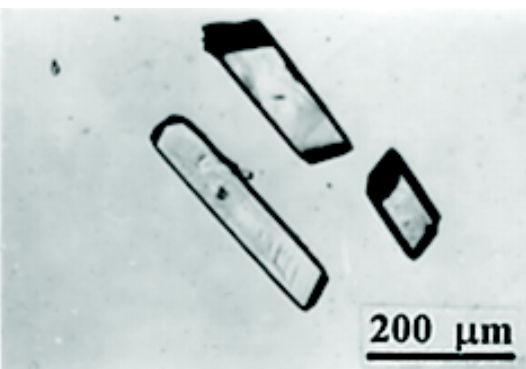

(b) Observed shape of paracetamol in Vapor

(001)

(0 $\overline{1} 1)$

(d) Observed shape of paracetamol in Water

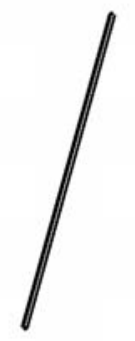

(f) Predicted shape of paracetamol in Cyclohexane

Figure 5.5: A comparison between predicted and observed [135] shapes of paracetamol under sublimation growth ((a):(b)) and when grown from aqueous solution ((c):(d)), indicating good agreement between ADDICT and experiments in both cases. Additionally, predicted shapes of paracetamol in methanol and cyclohexane are presented in (e) \& (f), respectively. 


\subsection{Case study: Lovastatin}

Lovastatin $\left(\mathrm{C}_{24} \mathrm{H}_{36} \mathrm{O}_{5}\right)$ is a drug that has been used to lower cholesterol, reducing the risk of cardiovascular diseases, $[13,137]$ and is another example of a non-centrosymmetric crystal system, with highly complicated bonding. Lovastatin crystallizes in the space group $P 2{ }_{1} 2{ }_{1}{ }_{1}$ with $\mathrm{Z}=1, \mathrm{~J}=1$ and the following lattice parameters: $a=22.15 \AA ; \mathrm{b}=17.32 \AA$; $\mathrm{c}=5.97 \AA$; and $\alpha=\beta=\gamma=90^{\circ}$ [13]. The relevant F-faces are $\{210\},\{101\},\{011\}$ and $\{020\}$. Figure 5.6 compares predicted growth shapes using ADDICT to experimentally observed shapes, for solution growth from isopropanol, methanol, acetone and ethyl acetate, where high-aspect ratio shapes are predicted. This comparison shows that the shapes predicted by ADDICT match experimental ones closely. Crystals from isopropanol and methanol are predicted to be needle-like (the broken lines in Figure 5.6 (a) and (b) indicate higher aspect ratios than can be displayed), while crystals from acetone and ethyl acetate are predicted to be rod-like with lower aspect ratio; this is confirmed by the experimental observations.

\subsection{Case study: D-mannitol $(\beta$ polymorph)}

D-mannitol is a renal diagnostic aid and its $\beta$ polymorph crystallizes in the space group $P 2{ }_{1}{ }_{1} 2{ }_{1}$ with $\mathrm{Z}=4, \mathrm{~J}=1$ and the following lattice parameters: $a=8.694 \AA ; \mathrm{b}=16.902 \AA$; $\mathrm{c}=5.549 \AA$; and $\alpha=\beta=\gamma=90^{\circ}$ [138]. Figure 5.7 compares ADDICT's prediction for aqueous crystal growth to experimental observations [139], which again confirm the success of the program. Instead of the rod-like habit from aqueous solution, ADDICT predicts a platelet morphology for sublimation growth (Figure 5.7 (b)). 


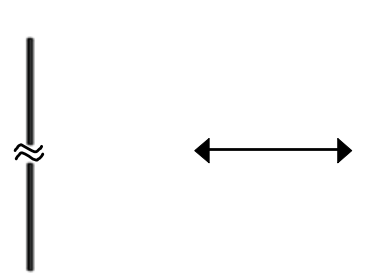

(a) Predicted shape of lovastatin in Isopropanol

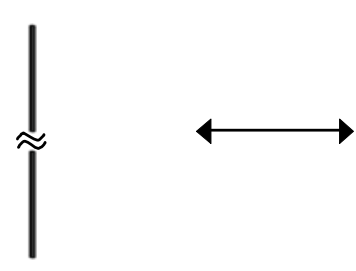

(c) Predicted shape of lovastatin in Methanol

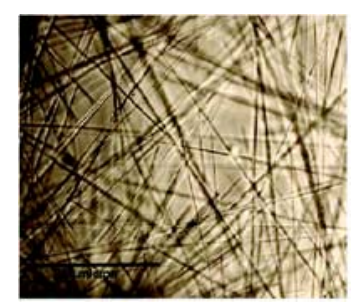

(b) Observed shape of lovastatin in Isopropanol

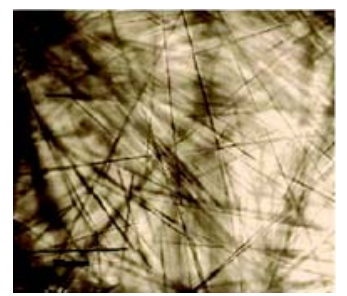

(d) Observed shape of lovastatin in Methanol

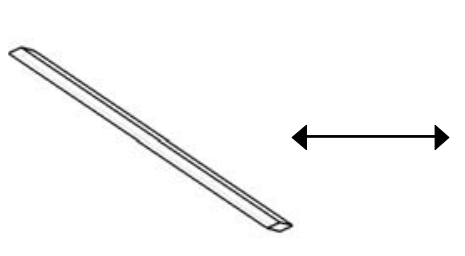

(e) Predicted shape of lovastatin in Acetone

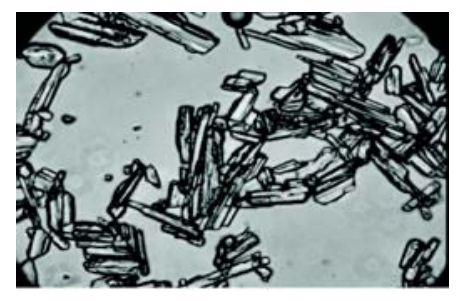

(f) Observed shape of lovastatin in Acetone

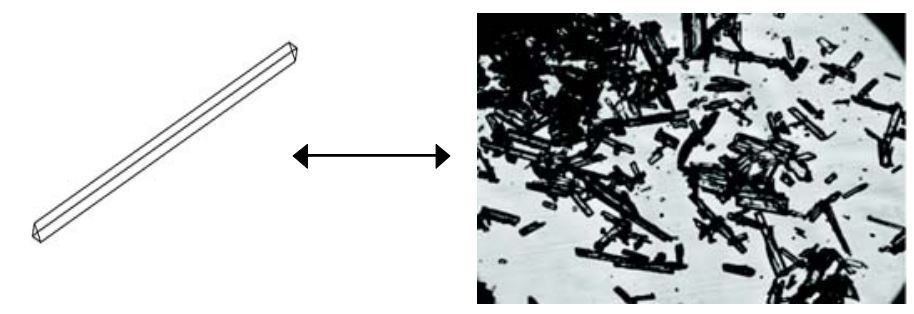

(g) Predicted shape of lovastatin in Ethyl acetate

(h) Observed shape of lovastatin in Ethyl acetate

Figure 5.6: A comparison between predicted (a), (c), (e), (g) and observed (b), (d), (f), (h) shapes of lovastatin when grown from isopropanol, methanol, acetone and ethyl acetate; ADDICT predicts needles in isopropanol and methanol and rods in acetone and ethyl acetate, matching experimental shapes. Images (b) and (d) are from Ref [13]; (f) and (h) are from a more recent determination within our laboratory. 


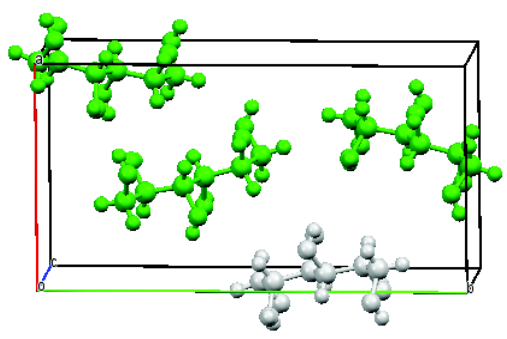

(a) Unit cell of D-mannitol

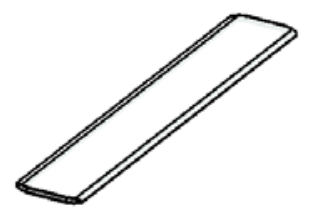

(b) Predicted in Vapor

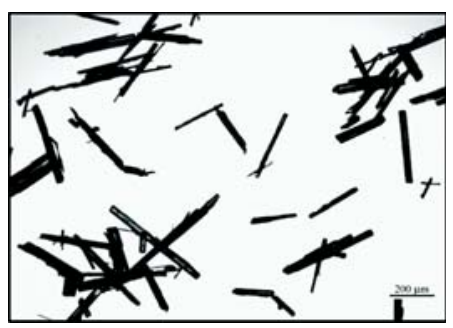

(d) Observed in Water

Figure 5.7: (a) The unit cell of D-mannitol (image produced with Mercury [39]); (b) predicted shapes of D-mannitol grown from vapor; (c) predicted and (d) observed [139] crystal shapes of D-mannitol grown from water.

\subsection{Case study: $\alpha$-Glycine}

The final example presented that compares predictions using ADDICT to experimental morphologies is the amino acid glycine, grown from an aqueous solution. Three polymorphs exist and $\alpha$-glycine will be considered here. The crystallization of $\alpha$-glycine has been successfully studied previously using a model based on the BCF mechanism [140]; morphology predictions obtained using ADDICT remains successful and remove the need for system-specific modeling expertise. 
The $\alpha$-glycine polymorph crystallizes in the monoclinic space group $P 2_{1} / n$ with lattice parameters: $\quad a=5.1054 \AA ; \mathrm{b}=11.9688 \AA ; \mathrm{c}=5.4645 \AA ; \alpha=\gamma=90^{\circ}$; and $\beta=111.697^{\circ} \quad$ [141]. Although $\mathrm{Z}=4$ and $\mathrm{J}=1$, a dimer growth unit is considered; the zwitterion interactions are, therefore, intra-dimer and not formed during crystal growth (the dimer is expected to exist in solution). Within the unit cell, the two dimer growth units are found at [uvw] positions $[0,0,0]$ and $[1 / 2,1 / 2,1 / 2]$. The families of $F$ faces for $\alpha$-glycine are $\{020\},\{110\}$ and $\{011\}$. The predicted crystal shape grown from water is shown in Figure 5.8 (b), which corresponds to the experimentally reported [142] result in Figure 5.8 (a). Detailed results including face specific parameters can be found in a previous paper [100].

(a)

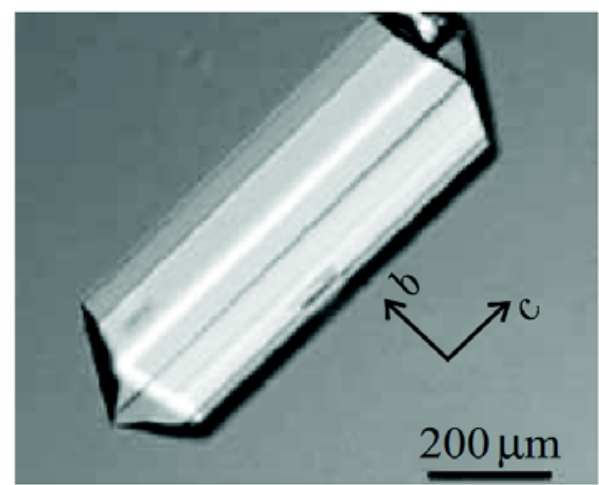

(b)

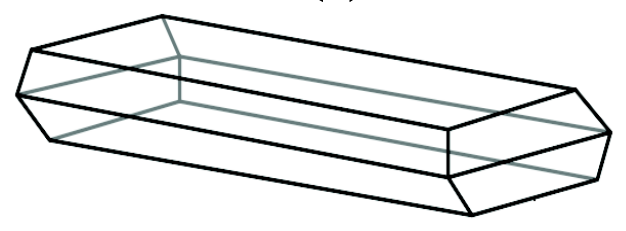

Figure 5.8: A comparison of the experimental shape of $\alpha$-glycine grown from water (a) [142] to the predicted shape using $\operatorname{ADDICT}(\mathrm{b})$. 


\section{Conclusions}

Controlling crystal growth to improve product functionality is highly desirable in every industry that manufactures or uses crystalline products. An engineering tool to screen through the available design space and find growth conditions that confer an optimum crystal morphology would, therefore, be extremely valuable. Mechanistic models for crystal growth have been established and developed, which serve as the foundation upon which such a predictive tool can be implemented. ADDICT serves as proof-of-concept, academic software that proves the feasibility and utility of these techniques. Many different organic compounds have been tested in ADDICT and results for naphthalene, anthracene, paracetamol, lovastatin, D-mannitol, and $\alpha$-glycine have been presented as an indication of the quality of predictions that can be obtained; the shapes predicted using ADDICT are in good agreement with the shapes that have been experimentally reported for both vapor and solution growth.

Additionally, ADDICT makes it possible to evaluate a wide variety of crystal materials quickly. As a result, it allows us to identify deficiencies in the methodology that are otherwise difficult to uncover and permits rapid testing of alternative approaches. These include: strategies to accurately describe stable/unstable edge configurations, alternative solvent effect formulations that better capture the underlying chemistry and supersaturation-dependent expressions for the kink density. These future changes could assimilate greater functionality or improved descriptions of real crystal systems into our mechanistic modeling. ADDICT only implements a model for spiral growth, so supersaturation-dependent shape effects that result from transitioning to different growth mechanisms (i.e. 2D nucleation \& growth) on certain face families can not currently be predicted using the model. Note that the kink rate for non-centrosymmetric molecules has a complex supersaturation-dependence that leads to relative growth rates that do depend on the 
supersaturation (albeit usually to a minor degree) even with all faces in the spiral regime. For centrosymmetric systems however, there is no dependence on supersaturation for the relative growth rates and hence crystal shapes. These limitations are likely acceptable for the controlled synthesis of pharmaceuticals, where low supersaturations and a spiral regime are applicable.

\section{Acknowledgment}

We are grateful for the financial support provided over many years by Rhodia, Merck, Eli Lilly, Pfizer, Bristol-Meyers-Squibb, Novartis and by the National Science Foundation (CBET-1159746).

\section{References}

(1) Schmidt C, Ulrich J. Morphology prediction of crystals grown in the presence of impurities and solvents-an evaluation of the state of the art. J Cryst Growth 2012; 353: 168-173.

(2) Wu C, Xie Y. Controlling phase and morphology of inorganic nanostructures originated from the internal crystal structure Chemical Communications. Chem Commun 2009; 28: 5943-57.

(3) Yang HG, Sun CH, Qiao SZ, Zou J, Liu G, Smith SC, Cheng HM, Lu GQ. Anatase TiO2 single crystals with a large percentage of reactive facets. Nature 2008; 453: 638-641.

(4) Variankaval N, Cote AS, Doherty MF. From form to function: crystallization of active pharmaceutical ingredients. AIChE J 2008; 54: 1682-1688.

(5) Winn D, Doherty MF. Modeling crystal shapes of organic materials grown from 
solution. AIChE J 2000; 46: 1348-1367.

(6) Yin JC, Zhou JS, Sun J, Qiu Y, Wei DZ, Shen YL. Study of the crystal shape and its influence on the anti-tumor activity of tumor necrosis factor-related apoptosis-inducing ligand (Apo2L/TRAIL). Cryst Res Technol 2008; 43: 888-893.

(7) Lovette M A, Doherty MF, Needle-shaped crystals: causality and solvent selection guidance based on periodic bond chains crystal. Cryst Growth Des 2013; 13: 33413352.

(8) Panina N, Van de Ven R. Janssen F, Meekes H, Vlieg E, Deroover G. Study of the needle-like morphologies of two $\beta$-Phthalocyanines. Cryst Growth Des 2009; 9: 840847.

(9) Freitas F, Sarmento V, Santilli C, Pulcinelli S. Controlling the growth of zirconia needles precursor from a liquid crystal template. Colloid Surface A 2010; 353: 77-82.

(10) Giri G, Park S, Vosgueritchian M, Shulaker MM, Bao Z. High-mobility, aligned crystalline domains of TIPS-pentacene with metastable polymorphs through lateral confinement of crystal. Adv Mater 2014; 26: 487-493.

(11) Dandekar P, Kuvadia ZB, Doherty MF. Engineering crystal morphology Annual Review of Materials Research. Annu Rev Mater Res 2013; 43: 359-386.

(12) Lovette MA, Browning AR, Griffin DW, Sizemore JP, Snyder RC, Doherty MF. Crystal shape engineering. Ind Eng Chem Res 2008; 47:9812-9833.

(13) Kuvadia ZB, Doherty MF. Spiral growth model for faceted crystals of noncentrosymmetric organic molecules grown from solution. Cryst Growth Des 2011; 11: 2780-2802. 
(14) Burton WK, Cabrera N, Frank F, Philos T. The growth of crystals and the equilibrium structure of their surfaces. Philos T R Soc A 1951; 243: 299-358.

(15) Shim HM, Koo KK, Crystal morphology prediction of hexahydro-1,3,5-trinitro-1,3,5triazine by the spiral growth model. Cryst Growth Des 2014; 14: 1802-1810.

(16) Shim HM, Koo KK. Prediction of growth habit of $\beta$-cyclotetramethylenetetranitramine crystals by the first-principles models. Cryst Growth Des 2015; 15: 3983-3991.

(17) Shim HM, Kim HS, Koo KK. Molecular modeling on supersaturation-dependent growth habit of 1,1-Diamino-2,2-dinitroethylene. Cryst Growth Des 2015; 15: 1833-1842.

(18) Dandekar P, Doherty MF. A mechanistic growth model for inorganic crystals: solidstate interactions. AIChE J 2014; 60: 3707-3719.

(19) Dandekar P, Doherty MF. A mechanistic growth model for inorganic crystals: Growth mechanism. AIChE J 2014; 60:3720-3731.

(20) Stack A G, Raiteri P, Gale JD. Accurate rates of the complex mechanisms for growth and dissolution of minerals using a combination of rare-event theories. J Am Chem Soc 2011; 134: 11-14.

(21) Stack AG, Grantham MC. Growth rate of calcite steps as a function of aqueous calciumto-carbonate ratio: independent attachment and detachment of calcium and carbonate ions. Cryst Growth Des 2010; 10: 1409-1413.

(22) Fenter P, Kerisit S, Raiteri P, Gale JD. Is the calcite-water interface understood? Direct comparisons of molecular dynamics simulations with specular X-ray reflectivity data. J Phys Chem C 2013; 117: 5028-5042. 
(23) Raiteri P, Gale JD, Water is the key to nonclassical nucleation of amorphous calcium carbonate. J Am Chem Soc 2010; 132: 17623-17634.

(24) Raiteri P, Gale JD, Quigley D, Rodger PM. Derivation of an accurate force-field for simulating the growth of calcium carbonate from aqueous solution: a new model for the calcite water interface. J Phys Chem C 2010; 114: 5997-6010.

(25) Bracco JN, Grantham MC, Stack AG, Calcite growth rates as a function of aqueous calcium-to-carbonate ratio, saturation index, and inhibitor concentration: insight into the mechanism of reaction and poisoning by strontium. Cryst Growth Des 2012; 12 : 3540-3548.

(26) Gibbs, J. W. The Scientific Papers of J. Willard Gibbs, Vol. 1, Thermodynamics; Dover: New York, 1961.

(27) Mutaftschiev B. Nucleation Theory. In Handbook of Crystal Growth, Vol. 1a, Fundamentals, Thermodynamics and kinetics; Hurle, D. T. J., Ed.; North-Holland: Amster- dam, 1993; Chapter 4: Nucleation Theory, p. 187-247.

(28) Wulff GZ. Zur Frage der Geschwindigkeit des Wachstums und der Auflösung von Krystallflächen. Z Kristallogr 1901; 34: 449-530.

(29) Herring, C. Some Theorems on the Free Energies of Crystal Surfaces. Phys. Rev. 1951; 82: 87-93.

(30) Kern R. The Equilibrium Form of a Crystal. In Morphology of Crystals: Part A; Sunagawa, I., Ed.; Terra Scientific Publishing Company: Tokyo, 1987; Chapter 2, p. 77-206.

(31) Zhang Y, Doherty MF. Simultaneous prediction of crystal shape and size for solution 
crystallization. AIChE J 2004; 50: 2101-2112.

(32) Zhang Y, Sizemore JP, Doherty MF. Shape evolution of 3-dimensional faceted crystals. AIChE J 2006; 52: 1906-1915.

(33) Frank FC. On the kinematic theory of crystal growth and dissolution processes. In Growth and Perfection of Crystals, pp. 411-419. John Wiley, New York, 1958.

(34) Chernov A. The kinetics of the growth forms of crystals. Sov Phys Cryst 1963; 7: 728730.

(35) Bravais A. Études Crystallographic. (Paris: Gauthier Villars) 1866.

(36) Donnay J, Harker D. A new law of crystal morphology extending the law of Bravais. Am Mineralogist 1937; 22: 446-467.

(37) Friedel M. Etudes sur la loi de Bravais, Bulletin de la Societe Francaise. Mineralogique 1907;30:326-455.

(38) Giacovazzo C, Monaco HL, Artioli G, Viterbo D, Milanesio M, Gilli G, Gilli P, Zanotti G, Catti M. Fundamentals of crystallography. Third Edition. 2011 Oxford Univeristy Press Inc., New York.

(39) Macrae CF, Edgington PR, McCabe P, Pidcock E, Shields GP, Taylor R, Towler M, van de Streek J. Mercury: visualization and analysis of crystal structures. J Appl Crystallogr 2006; 39: 453-457.

(40) Bisker-Leib V, Doherty MF. Modeling the crystal shape of polar organic materials: prediction of urea crystals grown from polar and nonpolar solvents. Cryst Growth Des 2001; $1: 455-461$. 
(41) Brunsteiner M, Price S L. Morphologies of organic crystals: sensitivity of attachment energy predictions to the model intermolecular potential. Cryst Growth Des 2001; 1 : 447-453.

(42) Lin $\mathrm{CH}$, Gabas N, Canselier JP, Pèpe G. Prediction of the growth morphology of aminoacid crystals in solution I. $\alpha$-Glycine. J Cryst Growth 1998; 191: 791-802.

(43) Hartman P, Perdok W. On the relations between structure and morphology of crystals. I Acta Crystallogr 1955; 8: 49-52.

(44) Hartman P, Perdok W. On the relations between structure and morphology of crystals. II Acta Crystallogr 1955, 8, 521-524.

(45) Hartman, P.; Perdok, W. On the relations between structure and morphology of crystals. III Acta Crystallogr 1955, 8, 525-529.

(46) Frey M, Genovesio-Taverne JC. Fontecilla-Camps JC. Application of the periodic bond chain (PBC) theory to the analysis of the molecular packing in protein crystals. J Cryst Growth 1988;90: 245-258.

(47) Hartman P, Bennema P. The attachment energy as a habit controlling factor: I. Theoretical considerations. J Cryst Growth 1980; 49: 145-156.

(48) Beyer T, Day GM, Price SL. The prediction, morphology, and mechanical properties of the polymorphs of paracetamol. J Am Chem Soc 2001; 123: 5086-5094.

(49) Anghel AT, Day GM, Price SL. A study of the known and hypothetical crystal structures of pyridine: Why are there four molecules in the asymmetric unit cell? Cryst Eng Comm 2002; 4: 348-355. 
(50) Coombes DS, Catlow RA, Gale JD, Rohl AL, Price SL. Calculation of attachment energies and relative volume growth rates as an aid to polymorph prediction. Cryst Growth Des 2005; 5: 879-885.

(51) Van der Voort E. The morphology of succinic acid crystals: the role of solvent interaction. J Cryst Growth 1991; 110: 662-668.

(52) Wang Z, Jiang P, Dang L. The morphology prediction of lysozyme crystals deduced from the BFDH law and attachment energy model based on the intermolecular interaction. 2010 4th International Conference on Bioinformatics and Biomedical Engineering. 2010; pp 1-4.

(53) Hartman P. In Crystal Growth: an Introduction; Hartman, P., Ed.; North Holland, Amsterdam, 1973; Chapter Structure and morphology, pp 367-402.

(54) Grimbergen RFP, Meekes H, Bennema P, Strom CS, Vogels LJP. On the prediction of crystal morphology. I. The Hartman-Perdok Theory Revisited. Acta Crystallogr 1998; 54: 491-500.

(55) Walker EM, Roberts KJ, Maginn SJ. A molecular dynamics study of solvent and impurity interaction on the crystal habit surfaces of $\epsilon$-caprolactam. Langmuir 1998; 14: 5620-5630.

(56) Lu J, Ulrich J. An improved prediction model of morphological modifications of organic crystals induced by additives. Cryst Res Technol 2003, 38, 63-73.

(57) Hammond RB, Pencheva K, Ramachandran V, Roberts KJ. Application of grid-based molecular methods for modeling solvent-dependent crystal growth morphology: aspirin crystallized from aqueous ethanolic solution. Cryst Growth Des 2007; 7: 1571-1574.

(58) Chen J, Trout BL. Computer-aided solvent selection for improving the morphology of needle-like crystals: a case study of 2,6-dihydroxybenzoic acid. Cryst Growth Des 2010; 
10: 4379-4388.

(59) Yang L, Dong Y. Crystal morphology study of N,N'-diacetylchitobiose by molecular dynamics simulation. Carbohyd Res 2011; 346: 2457-2462.

(60) Yi Q, Chen J, Le Y, Wang J, Xue C, Zhao H. Crystal structure and habit of dirithromycin acetone solvate: A combined experimental and simulative study. J Cryst Growth 2013; 372: 193-198.

(61) Chan EJ, Tejwani R, Derdour L. Use of molecular simulation in calculating a characteristic relative growth effect curvature to correlate factors influencing crystalline growth and other properties. Cryst Growth Des 2015; 15: 5754-5766.

(62) Boerrigter S, Josten G, Van De Streek J, Hollander F, Los J, Cuppen H, Bennema P, Meekes H. MONTY: Monte Carlo crystal growth on any crystal structure in any crystallographic orientation; application to fats. J Phys Chem A 2004; 108: 5894-5902.

(63) Boerrigter S, Cuppen H, Ristic R, Sherwood J, Bennema P, Meekes H. Explanation for the supersaturation-dependent morphology of monoclinic paracetamol. Cryst Growth Des 2002; 2: 357-361.

(64) Kossel W, Zur Theorie des Kristallwachstums. Nachrichten von der Gesellschaft der Wissenschaften zu G“ottingen. Mathematisch-Physikalische Klasse 1927; 1927: 135-143.

(65) Stranski IN, Zur theorie der kristallwachstums. Z Phys Chem 1928; 136: 259-78.

(66) Zhang J, Nancollas GH. Kink density and rate of step movement during growth and dissolution of an AB crystal in a nonstoichiometric solution. J Colloid Interf Sci 1998; 200: $131-145$.

(67) Vekilov PG. What determines the rate of growth of crystals from solution? Cryst Growth Des 2007; 7: 2796-2810. 
(68) Otálora F, García-Ruiz J. Nucleation and growth of the Naica giant gypsum crystals. Chem Soc Rev 2014; 43: 2013-2026.

(69) Chernov AA, Rashkovich LN, De Yoreo JJ. ABC of kink kinetics and density in a complex solution. AIP Conf P 2007; 916: 34-47.

(70) Frenkel J. On the surface motion of particles in crystals and the natural roughness of crystalline faces. J Phys USSR 1945; 9: 392-398.

(71) Lovette MA, Doherty MF, Multisite models to determine the distribution of kink sites adjacent to low-energy edges. Phys. Rev. E 2012; 85: 021604.

(72) Elwell D, Scheel H. Crystal Growth from High-Temperature Solutions; Academic Press, London, 1975.

(73) Kuroda T. Growth of a crystal surface with non-uniformity in supersaturation due to laminar flow of solution along the surface. J Cryst Growth 1985; 71: 84-94.

(74) Dirksen J, Ring T. Fundamentals of crystallization: kinetic effects on particle size distributions and morphology. Chem Eng Sci 1991; 46: 2389-2427.

(75) Chen K, Vekilov PG. Evidence for the surface-diffusion mechanism of solution crystallization from molecular-level observations with ferritin. Phys Rev E 2002; 66: 021606.

(76) Tilbury C, Daniel G, Will M, Doherty MF. Predicting the effect of solvent on crystal habit. Submitted.

(77) Chernov AA. Modern Crystallography III. Crystal Growth; Springer-Verlag: Berlin, 1984.

(78) Dandekar P, Doherty MF. Imaging Crystallization. Science 2014; 344: 705-706. 
(79) Hayashi Y, Maruyama M, Yoshimura M, Okada S, Yoshikawa HY, Sugiyama S, Adachi H, Matsumura H, Inoue T, Takano K, MurakamiS, Mori Y. Spiral growth can enhance both the normal growth rate and quality of tetragonal lysozyme crystals grown under a forced solution flow. Cryst Growth Des 2015; 15: 2137-2143.

(80) Land TA, De Yoreo JJ. The evolution of growth modes and activity of growth sources on canavalin investigated by in situ atomic force microscopy. J Cryst Growth 2000; 208: 623-637.

(81) Teng HH, Dove PM, De Yoreo JJ. Kinetics of calcite growth: surface processes and relationships to macroscopic rate laws. Geochimica et Cosmochimica Acta 2000; 64: 2255-2266.

(82) Markov I. Crystal Growth for Beginners: Fundamentals of Nucleation, Growth and Epitaxy. 2003.

(83) Uwaha M. Chapter 8: Growth kinetics: basics of crystal growth mechanisms. Handbook of Crystal Growth, Second Edition, Vol. I, Fundamentals: Thermodynamics and Kinetics, and Transport and Stability. Nishinaga T, editor. Elsevier B.V., 2014; 359-399.

(84) Wilcox WR. Transport phenomena in crystal growth from solution. Prog Cryst Growth Charact Mater 1993; 26: 153-194.

(85) Cubillas P, Anderson MW. Chapter 1: Synthesis mechanism: crystal growth and nucleation. In Zeolites and Catalysis: Synthesis, Reactions, and Applications eds Čjka J, Corma A, Zones S. Wiley-VCH (2010).

(86) Volmer M. Z Phys. Über gerichtetes Kristallwachstum (Ordered crystal growth) 1922, 9, 193-196. 
(87) Lovette MA, Doherty MF. Predictive modeling of supersaturation-dependent crystal shapes. Cryst Growth Des 2012; 12: 656-669.

(88) Nielsen AE. Kinetics of Precipitation; Pergamon Press Limited: Oxford, 1964.

(89) Lewis B. The growth of crystals of low supersaturation. J Cryst Growth 1974; 21: 29-39.

(90) Cuppen H, Meekes H, Van Enckevort W, Vlieg E. Birth-and-spread growth on the Kossel and a non-Kossel surface. J Cryst Growth 2006; 286: 188-196.

(91) Gilmer GH, Bennema P. Simulation of Crystal Growth with Surface diffusion. J Appl Phys 1972; 43: 1347-1360.

(92) Gilmer GH. Growth on perfect crystal faces. J Cryst Growth 1976; 36: 15-28.

(93) Cuppen HM, Van Veenendaal E, Van Suchtelen J. Van Enckevort WJP, Vlieg E. A Monte Carlo study of dislocation growth and etching of crystals. J Cryst Growth 2000; 219: 165-175.

(94) Rak M, Izdebski M, Brozi A. Kinetic Monte Carlo study of crystal growth from solution. Comput Phys Commun 2001; 138: 250-263.

(95) Piana S, Reyhani M, Gale JD. Simulating micrometre-scale crystal growth from solution. Nature 2005; 438: 70-73.

(96) Deij MA, Cuppen HM, Meekes H, Vlieg E. Steps on surfaces in modeling crystal growth. Cryst Growth Des 2007; 7: 1936-1942.

(97) Falo F, Bishop AR, Lomdahl PS, Horovitz B. Langevin molecular dynamics of interfaces: Nucleation versus spiral growth. Phys. Rev. B 1991; 43: 8081-8088.

(98) Piana S, Gale JD. Understanding the barriers to crystal growth: dynamical simulation of the dissolution and growth of urea from aqueous solution. J Am Chem Soc 2005; 78 
127: $1975-1982$.

(99) Piana S, Jones F, Gale JD. Assisted desolvation as a key kinetic step for crystal growth. J Am Chem Soc 2006; 128: 13568-13574.

(100) Snyder RC, Doherty MF. Predicting crystal growth by spiral motion. P Roy Soc AMath Phy 2009; 465: 1145-1171.

(101) Chernov A, Rashkovich L, Vekilov P. Steps in solution growth: dynamics of kinks, bunching and turbulence. J Cryst Growth 2005; 275: 1-18

(102)Wang J, Wolf RM, Caldwell JW, Kollman PA, Case DA. Development and testing of general amber force field. J Comput Chem 2004; 25: 1157-1174.

(103) Weiner PK, Kollman PA. AMBER: Assisted model building with energy refinement. A general program for modeling molecules and their interactions. $\mathrm{J}$ Comput Chem $1981 ; 2: 287-303$.

(104) Cuppen H, Meekes H, Van Enckevort W, Vlieg E, Knops H. Nonequilibrium free energy and kinetic roughening of steps on the Kossel (001) surface. Phys Rev B 2004; 69: 245404.

(105) Bennema P, Meekes H, Lui XY, De Yoreo JJ. Two centuries of morphology of crystals: integration of principles of mathematical crystallography, statistical mechanics of surface models and chemistry. Nanoscale Structure and Assembly at Solid-Fluid Interfaces, 2004; Volume I: Interfacial Structures versus Dynamics; 177208.

(106) Onsager L, A two-dimensional model with an order-disorder transition. Phys Rev 1944; 65: 117. 
(107) Dawson IM, Vand V. The observation of spiral growth-steps in n-Paraffin single crystals in the electron microscope. P Roy Soc Lond A-Math Phys 1951; 206: 555-562.

(108) Paloczi GT, Smith BL, Hansma PK, Walters DA, Wendman MA. Rapid imaging of calcite crystal growth using atomic force microscopy with small cantilevers. Appl Phys Lett 1998; 73:1658-1660.

(109) Teng HH, Dove PM, Orme CA, De Yoreo JJ. Thermodynamics of calcite growth: baseline for understanding biomineral formation. Science 1998;282: 724-727.

(110) Lovette MA, Doherty MF. Reinterpreting edge energies calculated from crystal growth experiments. J Cryst Growth 2011; 327: 117-126.

(111) Voronkov VV. Dislocation mechanism of growth with a low kink density. Sov Phys Cryst 1973; 18: 19-223.

(112) Kim S H, Dandekar P, Lovette MA, Doherty MF. Kink rate model for the general case of organic molecular crystals. Cryst Growth Des 2014; 14: 2460-2467.

(113) Eyring H. The activated complex in chemical reactions. J Chem Phys 1935; 3: 107-115.

(114) Salvalaglio M, Vetter T, Mazzotti M, Parrinello M. Controlling and predicting crystal shapes: the case of urea. Angew Chem Int Edit 2013; 52: 13369-13372.

(115) Kuvadia ZB, Doherty MF. Effect of structurally similar additives on crystal habit of organic molecular crystals at low supersaturation. Cryst Growth Des 2013; 13: 14121428.

(116) Van Oss C, Chaudhury M, Good R. Monopolar surfaces. Adv Colloid Interfac 1987; 28: 35-64. 
(117) Van Oss C, Good R, Chaudhury M. Additive and nonadditive surface tension components and the interpretation of contact angles. Langmuir 1988; 4: 884-891.

(118) Dunitz JD, Gavezzotti A. How molecules stick together in organic crystals: weak intermolecular interactions. Chem Soc Rev 2009; 38:2622-2633.

(119) Hansen CM. Hansen solubility parameters: a user's handbook CRC press, 2007.

(120) Beerbower A. Surface free energy: a new relationship to bulk energies. J Colloid Interf Sci 1971;35: 126-132.

(121) Burrell H. The challenge of the solubility parameter concept. J Paint Technol 1968; 40: $197-208$.

(122) Van Oss CJ. Interfacial forces in aqueous media. CRC press, 2006.

(123) Frisch, M.; Trucks, G.; Schlegel, H. B.; Scuseria, G.; Robb, M.; Cheeseman, J.; Scalmani, G.; Barone, V.; Mennucci, B.; Petersson, G.; Gaussian 09, 2009; 19: 227238.

(124) Wang J, Wang W, Kollman P A, Case DA. Antechamber: an accessory software package for molecular mechanical calculations. J Chem Inf Comp Sci 2001; 222: p.U403.

(125) Leach AR. Molecular modelling: principles and applications. Pearson Education, 2001.

(126) Lovette MA, Muratore M, Doherty MF. Crystal shape modification through cycles of dissolution and growth: Attainable regions and experimental validation. AIChE J 2012; 58: $1465-1474$.

(127) Brock CP, Dunitz JD. Temperature dependence of thermal motion in crystalline naphthalene. Acta Crystallogr B 1982; 38: 2218-2228. 
(128) Grimbergen RFP, Reedijk MF, Meekes H, Bennema P. Growth behavior of crystal faces containing symmetry-related connected nets: a case study of naphthalene and anthracene. J Phys Chem B 1998; 102: 2646-2653.

(129) Mason R. The crystallography of anthracene at 95K and 290K. Acta Cryst. 1964; 17: $547-555$.

(130) Docherty R, Roberts KJ. Modelling the morphology of molecular crystals; application to anthracene, biphenyl and b-succinic acid. J Cryst Growth 1988; 88:159-168.

(131) Cuppen H M, Graswinckel WS, Meekes WS. Screw dislocations on polycenes: a requirement for crystallization. Cryst Growth Des 2004; 4: 1351-1357.

(132) Smith KB, Bridson RH, Leeke GA. Crystallisation control of paracetamol from ionic liquids. Cryst Eng Comm 2014;16: 10797-10803.

(133) Lee T, Kuo CS, Chen YH. Solubility, polymorphism, crystallinity, and crystal habit of acetaminophen and ibuprofen. Pharm Technol 2006; 30: 72-92.

(134) Haisa M, Kashino S, Kawai R, Maeda H. The monoclinic form of $p$-Hydroxyacetanilide. Acta Crystallogr B 1976; 32: 1283-1285.

(135) Ristic R, Finnie S, Sheen D, Sherwood J. Macro- and micromorphology of monoclinic paracetamol grown from pure aqueous solution. J Phys Chem B 2001; 105: 9057-9066.

(136) Kuvadia ZB, Doherty MF. Reformulating multidimensional population balances for predicting crystal size and shape. AIChE J 2013; 59: 3468-3474.

(137) Chuong MC, Choy E, Douk K, Duong LH, Hoang SK, Le N, Lim M, Poirier, B, Prasad D, Radke M, Tran JQ, Williams DA. The development of delayed-then-extended-release lovastatin tablet. Int J Appl Pharm 2013; 5: 11-18. 
(138) Kaminsky W, Glazer AM, Crystal optics of D-mannitol, $\mathrm{C}_{6} \mathrm{H}_{14} \mathrm{O}_{6}$ : Crystal growth, structure, basic physical properties, birefringence, optical activity, Faraday effect, electro-optic effects and model calculations. Z Kristallogr 1997; 212:283-296.

(139) O'Sullivan B, Glennon B. Application of in situ FBRM and ATR-FTIR to the monitoring of the polymorphic transformation of D-mannitol. Org Process Res Dev 2005; 9: 884-889.

(140) Bisker-Leib V, Doherty MF. Modeling crystal shape of polar organic materials: applications to amino acids. Cryst Growth Des 2003; 3: 221-237.

(141) Jönsson PG, Kvick Å. Precision neutron diffraction structure determination of protein and nucleic acid components. III. The crystal and molecular structure of the amino acid $\alpha$-glycine. Acta Cryst 1972; B28: 1827-1833.

(142) Poornachary SK, Chow PS, Tan RBH, Davey RJ. Molecular speciation controlling stereoselectivity of additives: impact on the habit modification in a-glycine crystals. Cryst Growth Des 2007; 7: 254-261. 